\title{
Mitochondrial-Targeting Antioxidant SS-31 Suppresses Airway Inflammation and Oxidative Stress Induced by Cigarette Smoke
}

\author{
De-qing Yang $\mathbb{D}^{1},{ }^{1}$ Qiu-nan Zuo, ${ }^{2}$ Tao Wang, ${ }^{1}$ Dan Xu, ${ }^{1}$ Liu Lian, ${ }^{1}$ Li-juan Gao, ${ }^{1}$ Chun Wan, \\ Lei Chen, ${ }^{1}$ Fu-qiang Wen $\left(1,{ }^{1}\right.$ and Yong-chun Shen ${ }^{1}{ }^{1}$ \\ ${ }^{1}$ Division of Pulmonary Diseases, State Key Laboratory of Biotherapy of China and Department of Respiratory and Critical \\ Care Medicine, West China Hospital of Sichuan University, Chengdu 610041, China \\ ${ }^{2}$ Respiratory Ward, Department of Geriatrics, Sichuan Provincial People's Hospital, University of Electronic Science and Technology \\ of China, Chengdu 610072, China
}

Correspondence should be addressed to Fu-qiang Wen; wenfuqiang@scu.edu.cn and Yong-chun Shen; shen_yongchun@126.com Received 24 December 2020; Revised 21 March 2021; Accepted 26 April 2021; Published 15 June 2021

Academic Editor: Vinicius de Frias Carvalho

Copyright (C) 2021 De-qing Yang et al. This is an open access article distributed under the Creative Commons Attribution License, which permits unrestricted use, distribution, and reproduction in any medium, provided the original work is properly cited.

This study investigated whether the mitochondrial-targeted peptide SS-31 can protect against cigarette smoke- (CS-) induced airway inflammation and oxidative stress in vitro and in vivo. Mice were exposed to CS for 4 weeks to establish a CS-induced airway inflammation model, and those in the experimental group were pretreated with SS-31 $1 \mathrm{~h}$ before CS exposure. Pathologic changes and oxidative stress in lung tissue, inflammatory cell counts, and proinflammatory cytokine levels in bronchoalveolar lavage fluid (BALF) were examined. The mechanistic basis for the effects of SS-31 on CS extract- (CSE-) induced airway inflammation and oxidative stress was investigated using BEAS-2B bronchial epithelial cells and by RNA sequencing and western blot analysis of lung tissues. SS-31 attenuated CS-induced inflammatory injury of the airway and reduced total cell, neutrophil, and macrophage counts and tumor necrosis factor- (TNF-) $\alpha$, interleukin- (IL-) 6, and matrix metalloproteinase (MMP) 9 levels in BALF. SS-31 also attenuated CS-induced oxidative stress by decreasing malondialdehyde (MDA) and myeloperoxidase (MPO) activities and increasing that of superoxide dismutase (SOD). It also reversed CS-induced changes in the expression of mitochondrial fission protein (MFF) and optic atrophy (OPA) 1 and reduced the amount of cytochrome $c$ released into the cytosol. Pretreatment with SS-31 normalized TNF- $\alpha$, IL-6, and MMP9 expression, MDA and SOD activities, and ROS generation in CSE-treated BEAS-2B cells and reversed the changes in MFF and OPA1 expression. RNA sequencing and western blot analysis showed that SS-31 inhibited CS-induced activation of the mitogen-activated protein kinase (MAPK) signaling pathway in vitro and in vivo. Thus, SS-31 alleviates CS-induced airway inflammation and oxidative stress via modulation of mitochondrial function and regulation of MAPK signaling and thus has therapeutic potential for the treatment of airway disorders caused by smoking.

\section{Introduction}

Cigarette smoke (CS) contains thousands of toxins and is one of the most important risk factors for the development of chronic obstructive pulmonary disease (COPD), a progressive lung condition characterized by persistent airway inflammation and irreversible restriction of airflow $[1,2]$. COPD is a major health concern worldwide because of its high morbidity, mortality, and associated healthcare costs $[3,4]$. The pathogenesis of COPD is complex and is not fully understood. CS induces chronic airway inflammation, airway mucus hyperse- cretion, and oxidative stress, leading to clinically significant mechanical obstruction of small airways, reduced airflow, and a progressive decline in lung function $[5,6]$.

Mitochondria are the organelles responsible for energy metabolism and play an important role in maintaining cell function. Recent studies have suggested that CS can cause mitochondrial dysfunction and trigger inflammatory responses and oxidative stress, which are linked to COPD $[7,8]$. SS-31, a novel mitochondrial-targeting antioxidant compound, can eliminate reactive oxygen species (ROS) and increase ATP production in mitochondria, thus 
restoring mitochondrial membrane potential [9]. SS-31 was shown to protect cultured mouse microglial cells against lipopolysaccharide-induced inflammation and oxidative stress by stabilizing mitochondrial structure and reducing mitochondrial fission (FIS) 1 protein expression [10]. It also alleviated the inflammatory response and oxidative stress and exerted beneficial effects on leukocytes in type 2 diabetes patients [11]. In a mouse model of spinal cord injury-induced lung impairment, SS-31 attenuated mitochondrial dysfunction and inflammation and reduced the severity of lung damage [12]. However, it is unclear whether SS-31 can protect against CS-induced airway inflammation and oxidative stress.

In this study, we investigated the effect of SS-31 on inflammation and oxidative stress in the lung induced by CS in vitro and in vivo along with the underlying molecular mechanisms.

\section{Materials and Methods}

2.1. Animals. Male C57BL/6J mice (age 9-10 weeks, weighing 20-22 g) bred under specific pathogen-free conditions were purchased from GemPharmatech (Nanjing, Jiangsu, China) and housed at constant temperature of $23^{\circ} \mathrm{C} \pm 2^{\circ} \mathrm{C}$ and $50 \%$ $\pm 10 \%$ humidity on a $12: 12 \mathrm{~h}$ light/dark cycle (lights on from 6:00 a.m. to 6:00 p.m.). The mice had free access to food and water. Experimental procedures were conducted under aseptic conditions. Chambers and cages were cleaned every 3 days. The mice were handled in accordance with the ARRIVE guidelines developed by the National Center for the Replacement, Refinement, and Reduction of Animals in Research. The study protocol was reviewed and approved by the Animal Ethics Committee of West China Hospital, Sichuan University (approval no. 2020229A).

2.2. CS Exposure and Animal Treatment. Mice were divided into 4 groups: a control group $(n=7)$ exposed to room air without treatment, and 3 experimental groups exposed to CS for 75 min twice daily, 5 days per week for 4 weeks [13]. One experimental group (CS group, $n=7$ ) received no treatment; the SS-31 (L)+CS group $(n=8)$ was exposed to CS and treated with a low dose of SS-31 $(2.5 \mathrm{mg} / \mathrm{kg}$ once daily) refer to the slightly modified dosage [14]; and the SS-31 $(\mathrm{H})+\mathrm{CS}$ group $(n=7)$ was exposed to CS and treated with a high dose of SS-31 (5 mg/kg once daily) $[15,16]$. SS-31 (Topscience, Shanghai, China) was intraperitoneally injected $1 \mathrm{~h}$ before CS exposure $[13,17]$. After 4 weeks, all mice were sacrificed by intraperitoneal phenobarbital injection (Sigma-Aldrich, St. Louis, MO, USA) followed by exsanguination from the right ventricle and abdominal aorta. The heart was flushed with $10 \mathrm{~mL}$ sterile phosphate-buffered saline (PBS) from the right ventricle until the lungs turned white to collect bronchoalveolar lavage fluid (BALF) and lung tissue samples.

2.3. BALF Collection and Cell Counting. Right lungs were washed 3 times with $0.5 \mathrm{~mL}$ of sterile PBS, and $>1.3 \mathrm{~mL}$ of BALF was recovered from each mouse and centrifuged at $1000 \times g$ for $5 \mathrm{~min}$. The supernatant was stored at $-80^{\circ} \mathrm{C}$ for analysis of cytokine levels by enzyme-linked immunosorbent assay (ELISA). The cell pellet was resuspended in $0.2 \mathrm{~mL}$ PBS, and the total cell number was counted with a hemocytometer. Differential cell counting was performed by cytocentrifugation (Cytopro7620; Wescor, UT, USA) at $100 \times g$ for $10 \mathrm{~min}$ followed by Wright-Giemsa staining.

2.4. Histologic Examination of Lung Tissue. Left lungs without lavage were fixed with $4 \%$ phosphate-buffered paraformaldehyde under a constant pressure of $25 \mathrm{~cm} \mathrm{H}_{2} \mathrm{O}$ and embedded in paraffin; $4 \mathrm{~mm}$ thick sections were cut and stained with hematoxylin and eosin. An experienced pathologist who was blinded to the treatments scored lung inflammation based on the severity of lung lesions including peribronchiolar infiltrates, alveolar septal infiltrates, perivascular infiltrates, and combined bronchus-associated lymphoid tissue hyperplasia [13]. Giemsa staining was performed to assess the density of inflammatory cells in alveoli.

Alcian blue- (AB-) periodic acid Schiff (PAS) staining was performed to assess the levels of intracellular mucous glycoconjugates. Immunohistochemical staining for Muc5ac protein was performed using a kit (Santa Cruz Biotechnology, Santa Cruz, CA, USA). Briefly, lung sections were stained with anti-Muc5ac antibody (clone 45 M1, 1:100; Genetex, Irvine, CA, USA) and the percentage of the total airway epithelial area that was positive for $\mathrm{AB} / \mathrm{PAS}$ staining or anti-Muc5ac immunoreactivity was quantified using ImagePro Plus v6.0 software (Media Cybernetics, Bethesda, MD, USA).

2.5. CS Extract (CSE) Preparation. CSE was freshly prepared as previously described [18], with a few modifications. Briefly, the smoke of 6 Marlboro cigarettes was bubbled through $20 \mathrm{~mL}$ of Dulbecco's modified Eagle's medium (DMEM) prewarmed at $37^{\circ} \mathrm{C}$. The solution was passed through a $0.22 \mu \mathrm{m}$ filter after adjusting the $\mathrm{pH}$ to 7.4 , yielding $100 \%$ CSE. Serum-free DMEM was used to dilute the $100 \%$ CSE to the working concentrations.

2.6. Cell Culture and Treatments. BEAS-2B normal human bronchial epithelial cells (American Type Culture Collection, Manassas, VA, USA) were cultured in DMEM supplemented with $10 \%$ fetal bovine serum and $1 \%$ penicillin $G$ sodium/streptomycin sulfate (Invitrogen, Carlsbad, CA, USA). The effect of CSE on cell viability was evaluated with the Cell Counting Kit- (CCK-) 8 assay (Dojindo Laboratories, Tokyo, Japan). Cells $\left(5 \times 10^{3} /\right.$ well $)$ were seeded in 96-well plates and allowed to attach overnight, then incubated for $24 \mathrm{~h}$ in DMEM containing CSE (2\%, 4\%, 6\%, $8 \%$, and 10\%). After removing the supernatant, $10 \mu \mathrm{L}$ of CCK- 8 reagent in a serum-free medium ( $100 \mu \mathrm{L} /$ well) was added, followed by incubation for $1 \mathrm{~h}$ at $37^{\circ} \mathrm{C}$. Absorbance was measured on a spectrophotometer at $450 \mathrm{~nm}$.

To evaluate the role of the mitogen-activated protein kinase (MAPK) signaling pathway in the anti-inflammatory and antioxidant effects of SS-31, BEAS-2B cells were pretreated with the MAPK activator anisomycin $(20 \mu \mathrm{g} / \mathrm{mL} / \mathrm{mL})$ (APExBIO Technology, Houston, TX, USA) for $1 \mathrm{~h}$ [19]. The cells were divided into 5 groups: control, CSE (incubation with CSE for $24 \mathrm{~h}$ ), anisomycin+CSE (incubation with CSE 
for $24 \mathrm{~h}$ following pretreatment with $20 \mu \mathrm{g} / \mathrm{mL}$ anisomycin for $1 \mathrm{~h}$ ), anisomycin+SS-31+CSE group (treatment with $100 \mu \mathrm{M}$ SS-31 for $1 \mathrm{~h}$ followed by CSE for $24 \mathrm{~h}$ after pretreatment with $20 \mu \mathrm{g} / \mathrm{mL}$ anisomycin for $1 \mathrm{~h}$ ), and SS-31+CSE (incubation with CSE for $24 \mathrm{~h}$ following pretreatment with $100 \mu \mathrm{M}$ SS-31 for $1 \mathrm{~h}$ ). Cells or the supernatants were harvested for analyses. The dose of SS-31 and incubation time of CSE were selected based on previous studies [20-22].

2.7. Measurement of Inflammatory Cytokine Levels. BALF levels of interleukin- (IL-) 6 and tumor necrosis factor(TNF-) $\alpha$ were measured with ELISA kits for mice (NeoBioscience, Shenzhen, China) according to the manufacturers' instructions. The level of matrix metallopeptidase (MMP) 9 was measured with an ELISA kit for mice (R\&D Systems, Minneapolis, MN, USA) according to the manufacturer's instructions; the stated detection limits were $15 \mathrm{pg} \cdot \mathrm{mL}^{-1}$ for TNF- $\alpha$, $1.6 \mathrm{pg} \cdot \mathrm{mL}^{-1}$ for IL-6, and $0.014 \mathrm{ng} \cdot \mathrm{mL}^{-1}$ for MMP9. TNF- $\alpha$, IL-6, and MMP9 levels in culture supernatant were determined with ELISA kits for humans (NeoBioscience) according to the manufacturers' instructions; the stated detection limits were $7.8 \mathrm{pg} \cdot \mathrm{mL}^{-1}$ for TNF- $\alpha, 0.39 \mathrm{pg} \cdot \mathrm{mL}^{-1}$ for IL-6, and $15.6 \mathrm{pg} \cdot \mathrm{mL}^{-1}$ for MMP9.

2.8. Detection of Oxidative Stress and Intracellular ROS. Malondialdehyde (MDA), superoxide dismutase (SOD), myeloperoxidase (MPO) activities in mouse lung homogenate and glutathione peroxidase (GSH-Px), MDA, and SOD activities as well as intracellular ROS production in cells were determined according to commercial protocols (Nanjing Jiancheng Bioengineering Institute, Jiangsu, China).

2.9. RNA Sequencing. RNA sequencing was performed as previously described [23]. Briefly, total RNA from lung tissue of the CS and SS-31 $(\mathrm{H})+\mathrm{CS}$ groups was isolated and the quality was verified according to the protocols of Illumina (San Diego, CA, USA). A total of $2 \mu \mathrm{g}$ RNA per sample was used as input material for library construction. Strandspecific sequencing libraries were generated by the dUTP method with RNA obtained using the NEBNext Ultra Directional RNA Library Prep Kit (Illumina) according to the manufacturer's instructions. RNA sequencing was performed on an Illumina Hiseq 2000 platform by Genewiz (Suzhou, China), generating $100 \mathrm{bp}$ paired-end reads. Adapter sequences were removed from the raw sequencing data, and the individual libraries were converted to FASTQ format. Sequence reads were aligned to the mouse genome (mm10) with TopHat2 v2.0.9, and the resultant alignment files were reconstructed with Cufflinks v2.1.1 and Scripture (beta2). For mRNA analyses, the RefSeq database (Build 37.3) was used as the source of annotation references. The read counts of each transcript were normalized to the length of the individual transcript and to the total mapped fragment counts in each sample and expressed as fragments per kilobase of exon per million fragments of mRNA mapped in each sample. Differential expression analyses were conducted using only samples from the CS and SS-31 (H)+CS groups. $P<0.05$ was used as the cutoff for differentially expressed genes (DEGs).
2.10. Bioinformatic Analysis. Enrichment analysis of DEGs was carried out to detect overrepresented functional terms in the genomic background. Gene Ontology (GO) analysis of biological processes, cellular components, and molecular function was performed using the GO-seq R package [24]. Enriched DEG signaling pathway analyses were conducted using the Kyoto Encyclopedia of Genes and Genomes (KEGG) database.

2.11. Western Blot. Protein samples were isolated from the right lung of mice and from BEAS-2B cells with radioimmunoprecipitation assay lysis buffer supplemented with $1 \mathrm{mM}$ phenylmethanesulfonyl fluoride (Cell Signaling Technology, Danvers, MA, USA). Total protein was fractionated by $10 \%$ sodium dodecyl sulfate-polyacrylamide gel electrophoresis and transferred to a polyvinylidene difluoride membrane. After blocking with $5 \%$ bovine serum albumin in Trisbuffered saline at room temperature for $1 \mathrm{~h}$, the membrane was incubated overnight at $4^{\circ} \mathrm{C}$ with antibodies against optic atrophy (OPA) 1 (mouse antibody from Cell Signaling Technology; human antibody from Proteintech, Rosemont, IL, USA); mitochondrial fission factor (MFF) (mouse antibody from Cell Signaling Technology; human antibody from Proteintech); and extracellular signal-regulated kinase (ERK), phosphorylated- (P-) ERK, P38, P-P38, and glyceraldehyde 3-phosphate dehydrogenase (GAPDH) (all from Cell Signaling Technology). They were then incubated with horseradish peroxidase-conjugated secondary antibodies, and immune complexes were detected with SuperSignal West Pico chemiluminescent substrate (Pierce, Rockford, IL, USA).

Cytoplasmic proteins were extracted using the Cytoplasmic Protein Extraction Kit (KeyGEN BioTech, Nanjing, China), and mitochondrial proteins were extracted using the Mitochondria Isolation Kit (Beyotime Biotech, Shanghai, China). Cytoplasmic extracts were probed with antibodies against cytosolic cytochrome c (Genetex) and GAPDH (Cell Signaling Technology). Mitochondrial extracts were probed with antibodies against mitochondrial cytochrome c (Genetex) and cytochrome c oxidase (COX) IV (Proteintech). Each experiment was repeated 3 times with different mice. The signal intensity of protein bands was quantified using ImageJ software (National Institutes of Health, Bethesda, MD, USA).

2.12. Statistical Analysis. Data are expressed as mean \pm standard deviation, and group means were compared by one-way analysis of variance followed by the least significant difference test for multiple comparisons. Data were analyzed, and figures were prepared using Prism 7 software (GraphPad, San Diego, CA, USA). $P<0.05$ was considered statistically significant.

\section{Results}

3.1. SS-31 Reverses the CS-Induced Increases in Inflammatory Cell Numbers and Cytokine Release in Mouse Lung. Total cell, neutrophil, and macrophage counts in mouse BALF were increased in CS-exposed mice, which was abrogated by pretreatment with SS-31 (Figures 1(a)-1(c)). Meanwhile, 4 weeks of CS exposure increased the levels of IL-6, TNF- $\alpha$, 


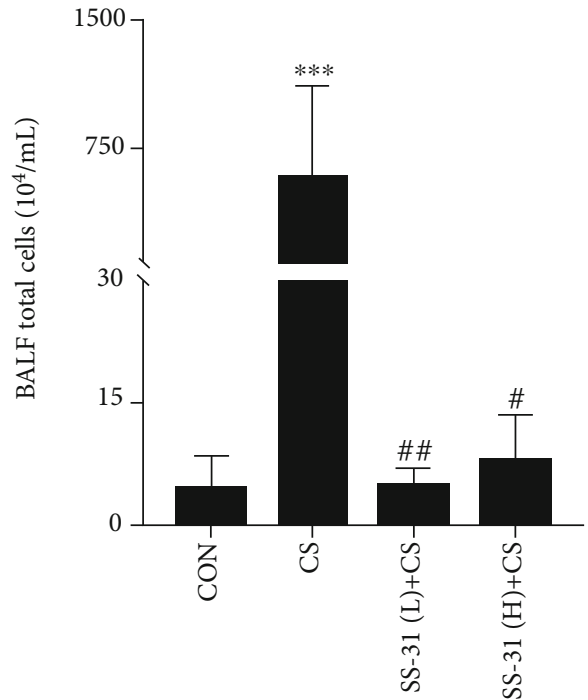

(a)

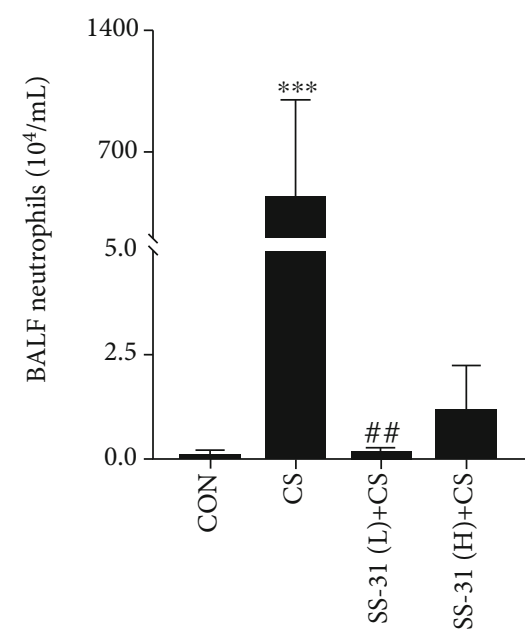

(b)

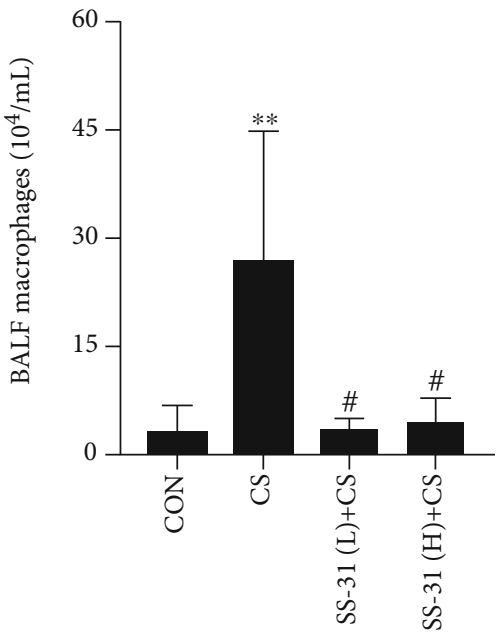

(c)

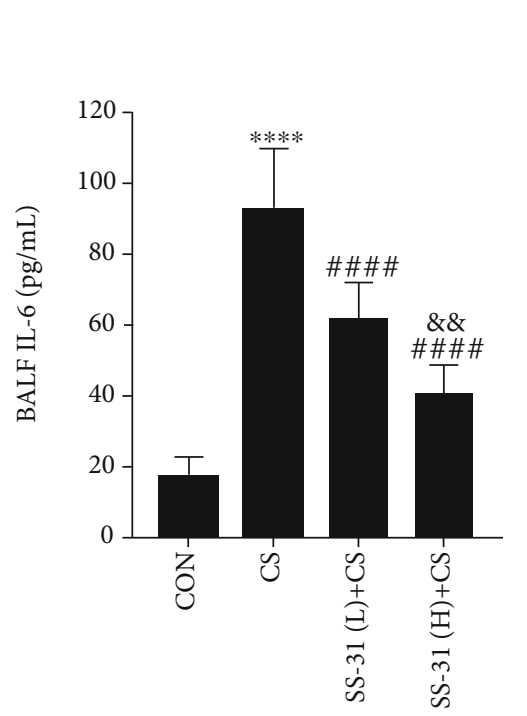

(d)

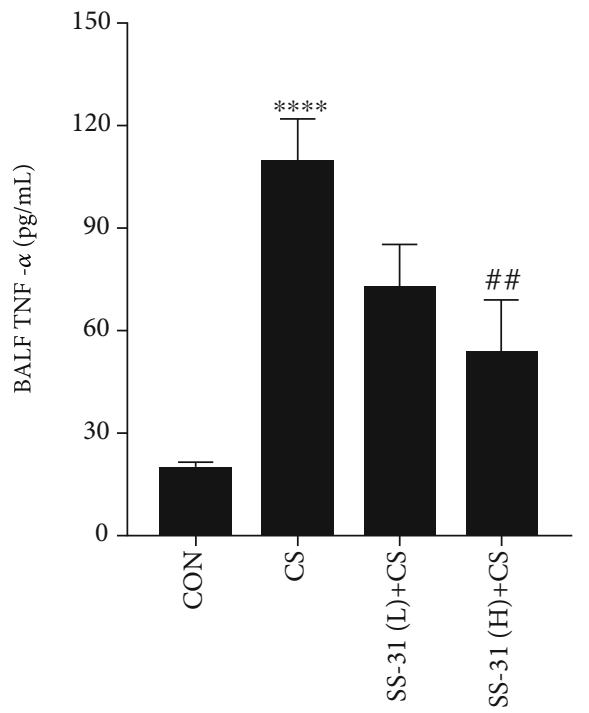

(e)

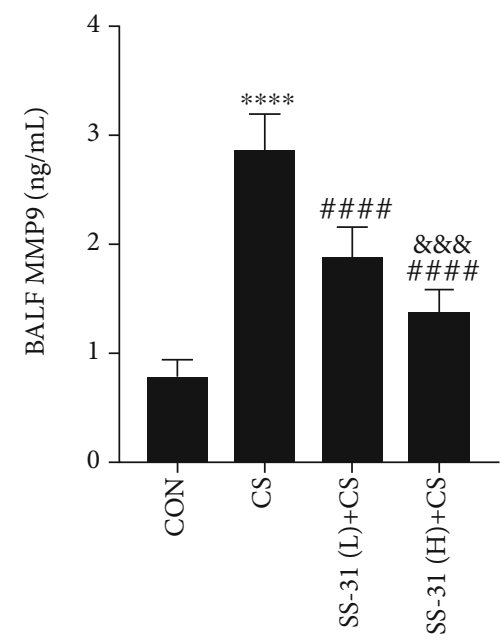

(f)

FIgURE 1: SS-31 reduces CS-induced increases in inflammatory cell numbers and cytokine infiltration in mouse lung. (a-f) Total cell (a), neutrophil (b), and macrophage (c) counts and IL-6 (d), TNF- $\alpha$ (e), and MMP9 (f) levels in mouse BALF (control: $n=7$, CS: $n=5$, SS-31 (L)+CS: $n=8$, SS-31 (H)+CS: $n=5) .{ }^{* *} P<0.01,{ }^{* * *} P<0.001$, and ${ }^{* * * *} P<0.0001$ vs. control; ${ }^{\#} P<0.05$, ${ }^{\# \#} P<0.01$, and ${ }^{\# \# \# \#} P<0.0001$ vs. CS; ${ }^{\& \&} P<0.01,{ }^{\& \& \&} P<0.001$, and SS-31 (L)+CS vs. SS-31 (H)+CS. Abbreviations: BALF: bronchoalveolar lavage fluid; CS: cigarette smoke; SS-31 (H): high-dose SS-31 (5 mg/kg); SS-31 (L): low-dose SS-31 (2.5 mg/kg).

and MMP9 in BALF, an effect that was abolished by SS-31 (Figures $1(\mathrm{~d})-1(\mathrm{f}))$.

\subsection{SS-31 Abrogates Histologic Changes in the Lung Induced} by CS. Four weeks of exposure to CS markedly increased peribronchial inflammatory cell infiltration, airway epithelial cell hyperplasia, airway epithelium thickening, and lumen obstruction by mucus and cell debris (Figures $2(\mathrm{~b})$ and $2(\mathrm{e}))$. These changes were abolished by low-dose $(2.5 \mathrm{mg} / \mathrm{kg})$ and high-dose $(5 \mathrm{mg} / \mathrm{kg})$ SS-31 pretreatment, with the latter yielding better results $(P<0.01$; Figure $2(\mathrm{e}))$. Giemsa staining showed that the number of neutrophils and macrophages was increased in CS-exposed mice, but this was reversed by SS-31 pretreatment (Figures 2(f)-2(i)).
3.3. SS-31 Suppresses CS-Induced Airway Mucus Hypersecretion. Mucus proteins were stained with AB/PAS. CS exposure significantly increased the secretion of airway mucus proteins (Figure 3), but this was suppressed by highdose SS-31 pretreatment. Accordingly, the airway mucus protein Muc5ac was upregulated after 4 weeks of CS exposure (Figures 4(b) and 4(e)) compared to the control group (Figure 4(a)), but this was abrogated by high-dose SS-31 (Figures 4(d) and 4(e)).

3.4. SS-31 Attenuates CS-Induced Oxidative Stress in Mouse Lung. After 4 weeks of CS exposure, MDA and MPO activities were increased (Figures 5(a) and 5(c)) whereas SOD activity was decreased (Figure 5(b)) in the lungs of mice, 

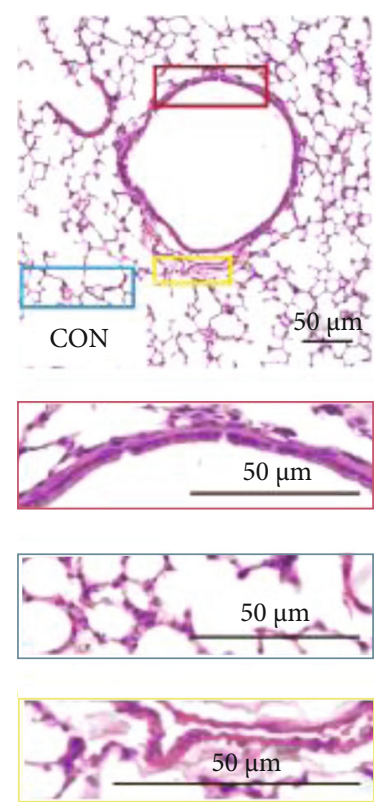

(a)
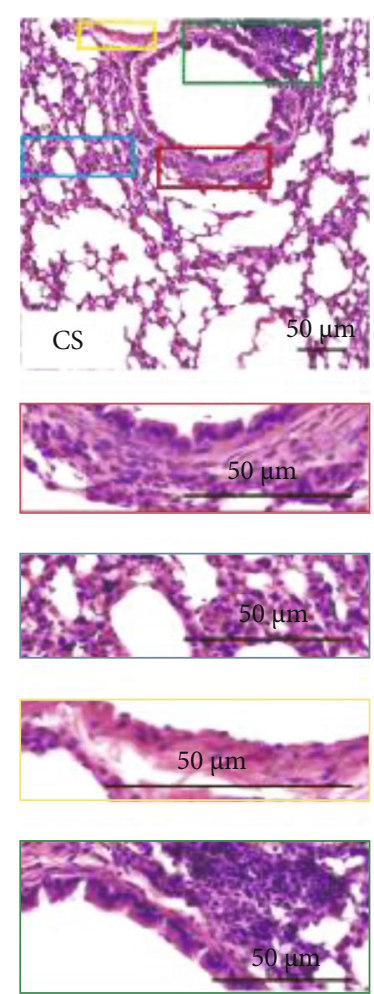

(b)
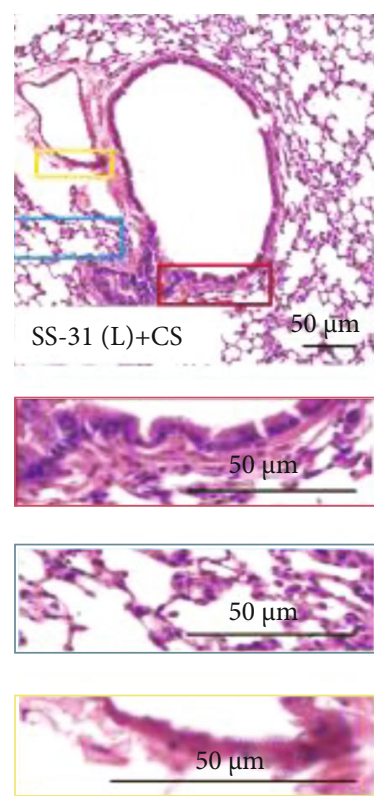

(c)
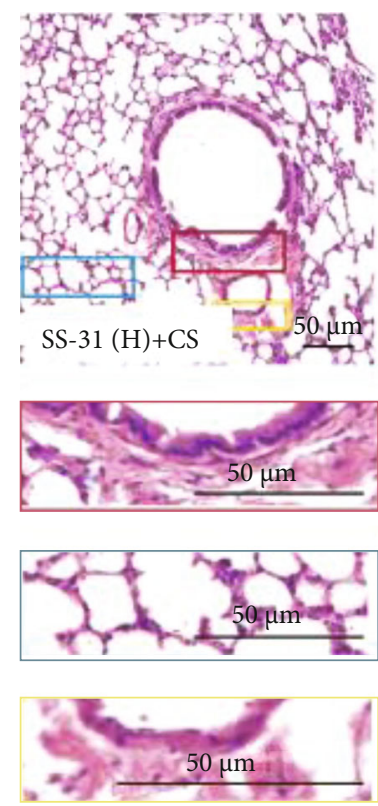

(d)

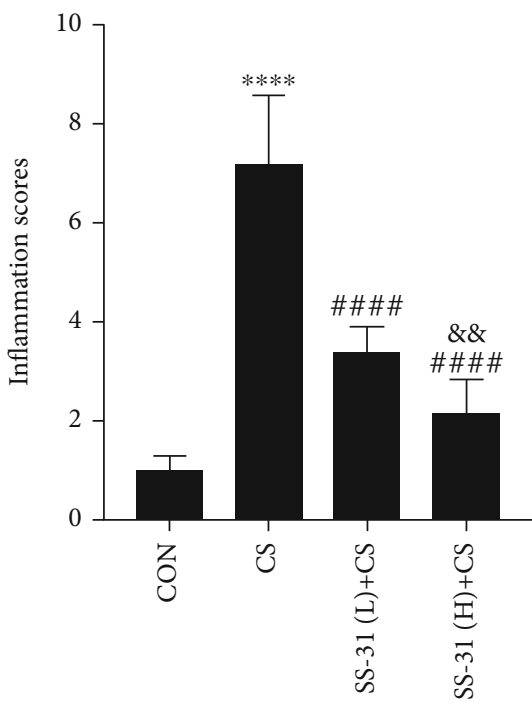

(e)

Figure 2: Continued. 


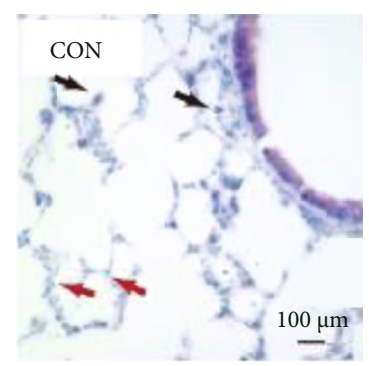

(f)

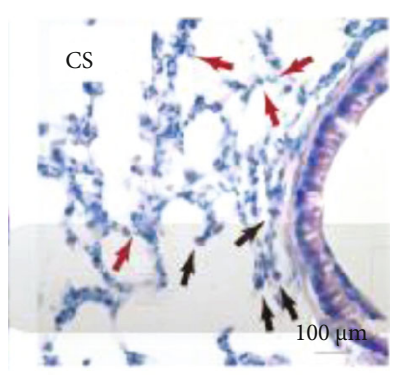

(g)

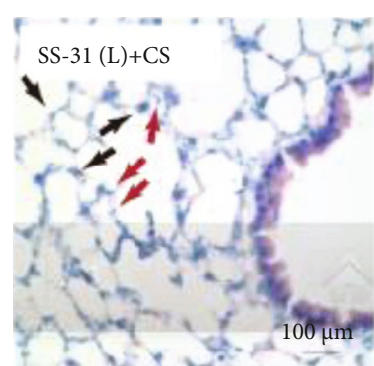

(h)

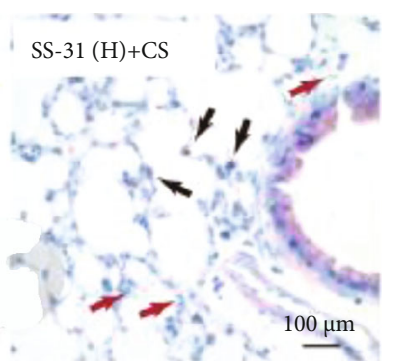

(i)

FIGURE 2: SS-31 attenuates CS-induced histologic changes in mouse lung. (a-d) H\&E staining of mouse lung tissue from control ( $n=7$ ) (a), CS $(n=7)(\mathrm{b})$, SS-31 (L)+CS $(n=8)(\mathrm{c})$, and SS-31 (H)+CS $(n=7)(\mathrm{d})$ groups (200x magnification, scale bar $=50 \mu \mathrm{m})$. (e) Inflammation scores of mouse lungs. Bottom images depict an enlarged view of boxed areas in corresponding top images; red box: peribronchiolar infiltrates; blue box: alveolar septal infiltrates; yellow box: perivascular infiltrates; green box: bronchus-associated lymphoid tissue hyperplasia. (f-i) Giemsa staining of mouse lung tissue from control (f), CS (g), SS-31 (L)+CS (h), and SS-31 (H)+CS (i) groups (600x magnification, scale bar $=100 \mu \mathrm{m})$. Red arrow: neutrophils; black arrow: macrophages. ${ }^{* * * *} P<0.0001$ vs. control; ${ }^{\# \# \# \# ~} P<0.0001$ vs. CS; ${ }^{\& \&} P<0.01$ SS-31 (L)+CS vs. SS-31 (H)+CS. Abbreviations: CS: cigarette smoke; H\&E: hematoxylin and eosin; SS-31 (H): high-dose SS-31 $(5 \mathrm{mg} / \mathrm{kg}) ;$ SS-31 (L): low-dose SS-31 (2.5 mg/kg).
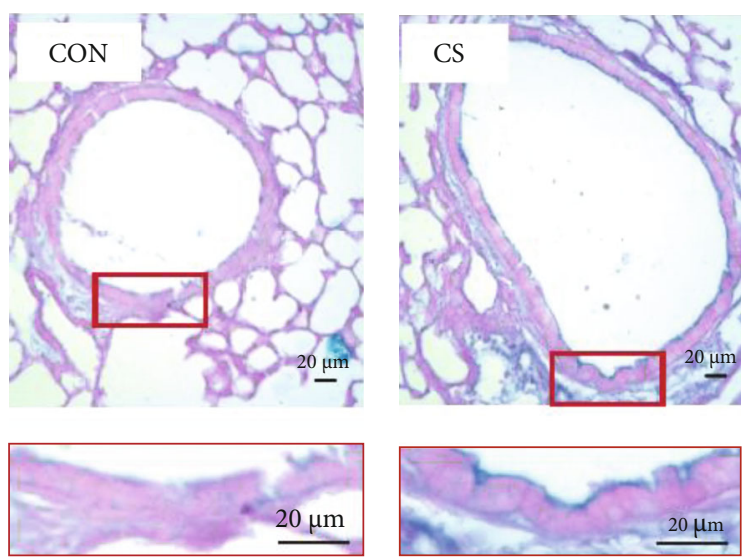

(a)

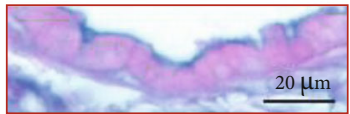

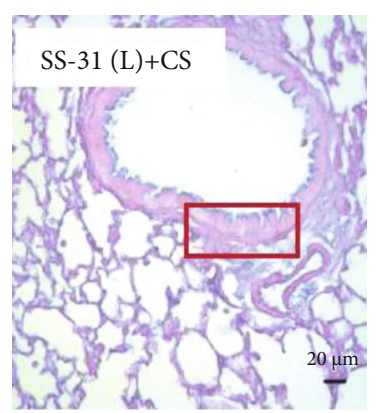

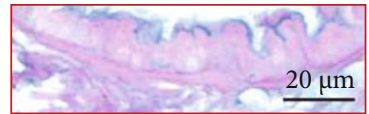

(c)

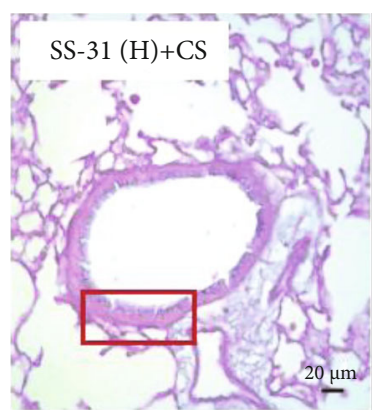

(b)

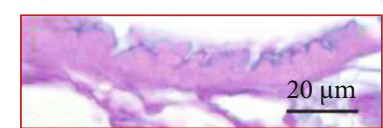

(d)

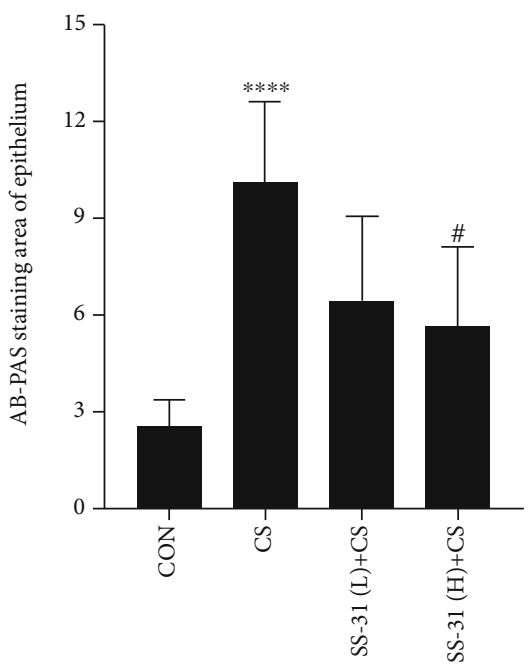

(e)

FIGURE 3: SS-31 attenuates CS-induced airway mucus hypersecretion. (a-d) Mouse lung tissue from control $(n=7)(\mathrm{a})$, CS ( $n=7)(\mathrm{b})$, SS-31 $(\mathrm{L})+\mathrm{CS}(n=8)(\mathrm{c})$, and SS-31 $(\mathrm{H})+\mathrm{CS}(n=7)(\mathrm{d})$ groups after AB/PAS staining (400x magnification, scale bar $=20 \mu \mathrm{m})$. (e) The positive percentage of epithelial area with AB/PAS staining. Bottom images depict enlarged views of boxed areas in corresponding top images. ${ }^{* * * *} P<0.0001$ vs. control; ${ }^{\#} P<0.05$ vs. CS. Abbreviations: AB/PAS: Alcian blue/periodic acid Schiff; CS: cigarette smoke; SS-31 (H): high-dose SS-31 (5 mg/kg); SS-31 (L): low-dose SS-31 (2.5 mg/kg). 


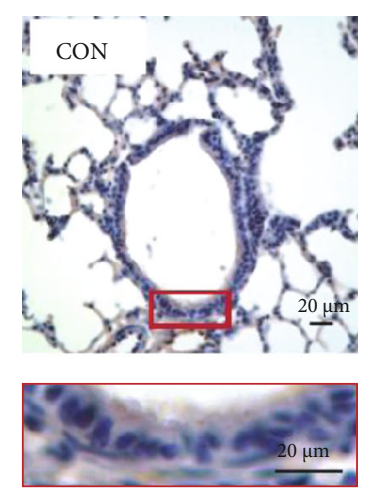

(a)
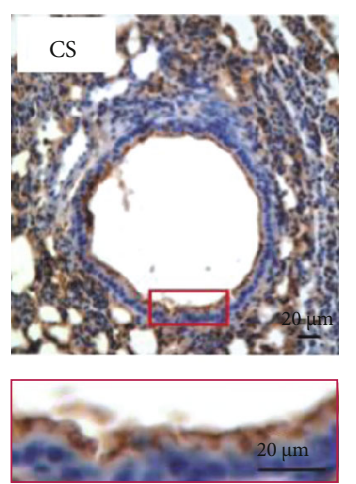

(b)

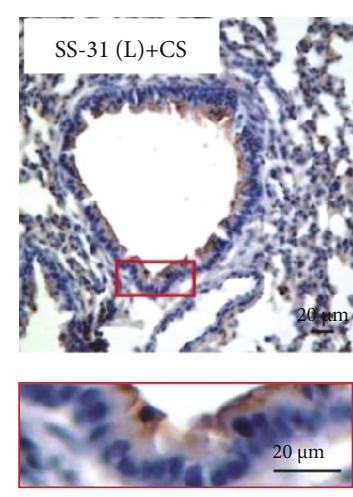

(c)
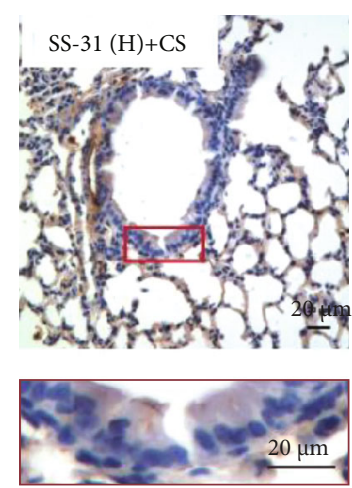

(d)

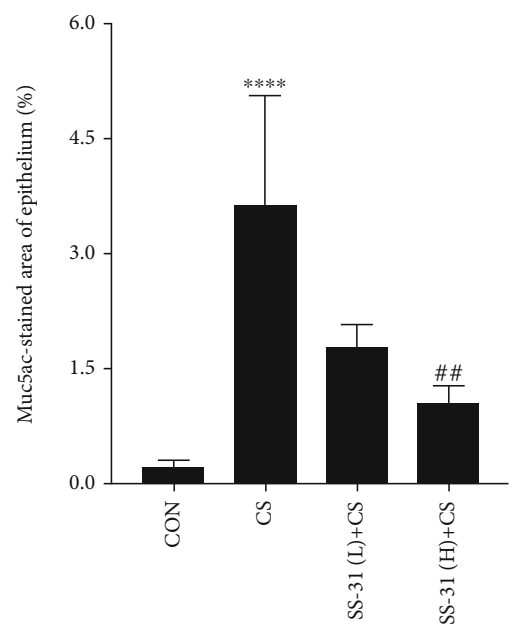

(e)

FIGURE 4: SS-31 suppresses CS-induced airway mucus protein secretion. (a-d) Immunohistochemical detection of Muc5ac in mouse airway epithelium (400x magnification, scale bar $=20 \mu \mathrm{m})$ from control (a), CS (b), SS-31 (L)+CS (c), and SS-31 (H)+CS (d) groups $(n=7$ per group). (e) The positive percentage of epithelial area with anti-Muc5ac immunoreactivity. Bottom images depict enlarged views of boxed areas in corresponding top images. ${ }^{* * * *} P<0.0001$ vs. control; ${ }^{\# \#} P<0.01$ vs. CS. Abbreviations: CS: cigarette smoke; SS-31 (H): high-dose SS-31 (5 mg/kg); SS-31 (L): low-dose SS-31 (2.5 mg/kg).

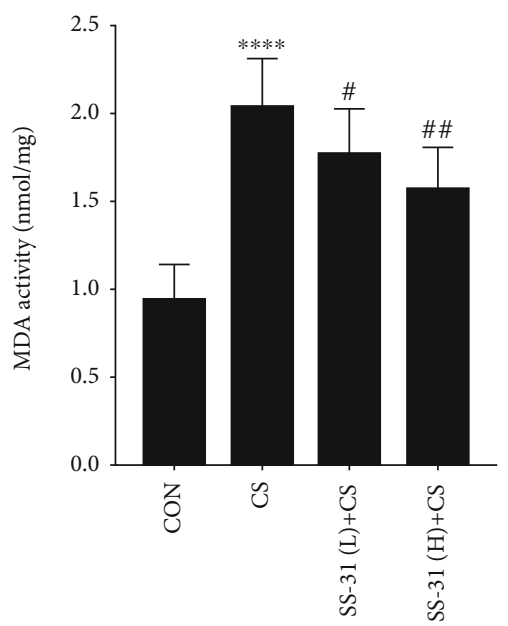

(a)

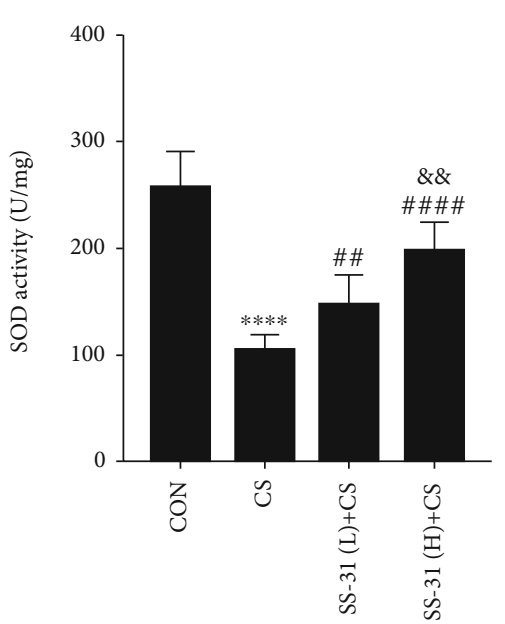

(b)

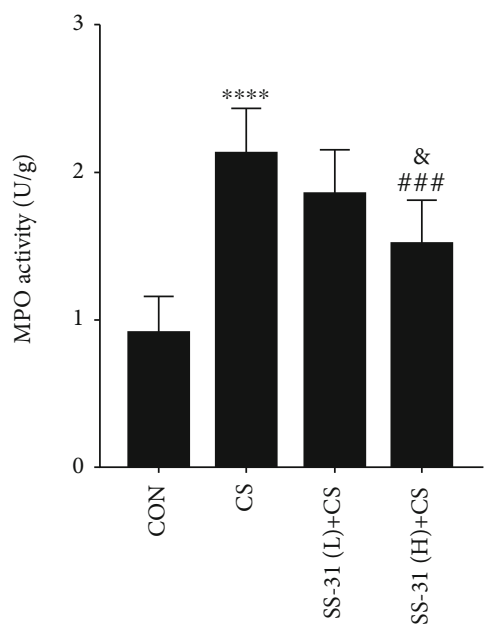

(c)

FIgURE 5: SS-31 attenuates CS-induced oxidative stress in mouse lung. (a-c) Effects of SS-31 on lung MDA (a), SOD (b), and MPO (c) activities in CS-exposed mice $\left(n=7\right.$ per group). ${ }^{* * * *} P<0.0001$ vs. control; ${ }^{\#} P<0.05,{ }^{\# \#} P<0.01$, ${ }^{\# \#} P<0.001$, and ${ }^{\# \# \# \#} P<0.0001$ vs. CS; ${ }^{\&} P<0.05,{ }^{\& \&} P<0.01$, and SS-31 (L)+CS vs. SS-31 (H)+CS. Abbreviations: CS: cigarette smoke; MDA: malondialdehyde; MPO: myeloperoxidase; SOD: superoxide dismutase; SS-31 (H): high-dose SS-31 (5 mg/kg); SS-31 (L): low-dose SS-31 (2.5 mg/kg). 

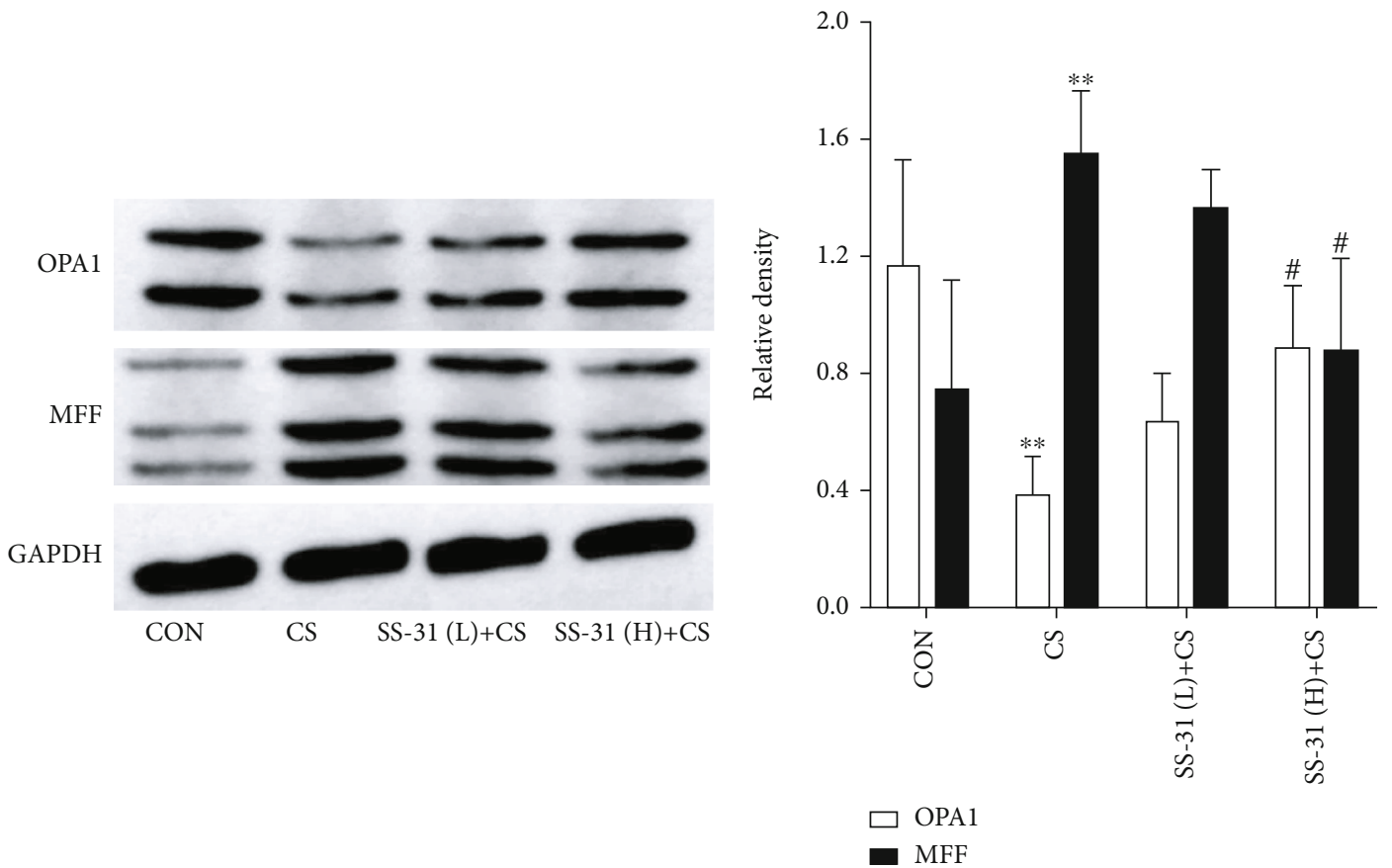

(a)

(b)
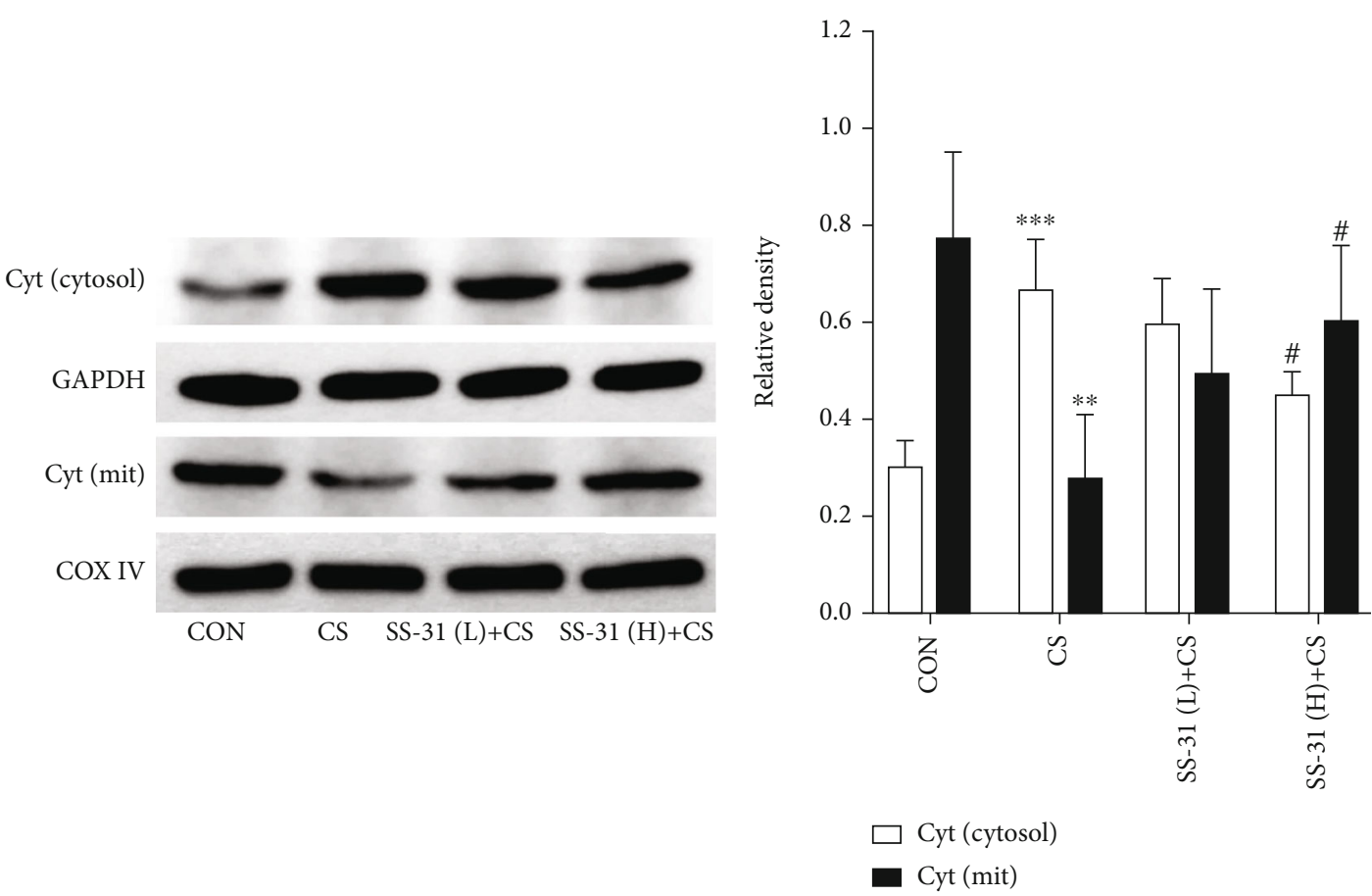

(c)

(d)

Figure 6: SS-31 prevents mitochondrial dysfunction in CS-induced mouse lung. (a-d) Protein levels of OPA1, MFF (a, b), and cytochrome c $(c, d)$ in lungs were evaluated by western blot following exposure to CS with or without SS-31 pretreatment. GAPDH or COX IV was used as a loading control ( $n=3$ per group). ${ }^{* *} P<0.01$ and ${ }^{* *} P<0.001$ vs. control; ${ }^{*} P<0.05$ vs CS. Abbreviations: COX IV: cytochrome c oxidase IV; CS: cigarette smoke; Cyt c: cytochrome c; GAPDH: glyceraldehyde 3-phosphate dehydrogenase; MFF: mitochondrial fission factor; mit: mitochondrion; OPA1: optic atrophy 1; SS-31 (H): high-dose SS-31 (5 mg/kg); SS-31 (L): low-dose SS-31 (2.5 mg/kg).

indicating that oxidative stress was induced. SS-31 pretreatment mitigated this effect in a dose-dependent manner (Figure 5): SOD and MPO activities were significantly higher in mice pretreated with $5 \mathrm{mg} / \mathrm{kg}$ SS-31 as compared to $2.5 \mathrm{mg} / \mathrm{kg}$ SS-31 before CS (Figures 5(b) and 5(c)), suggesting that SS-31 has strong antioxidant activity. MPO activity in 


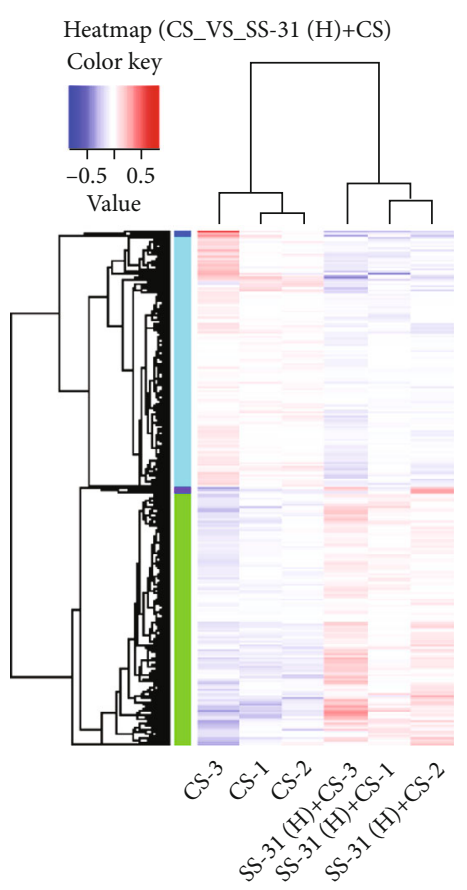

(a)

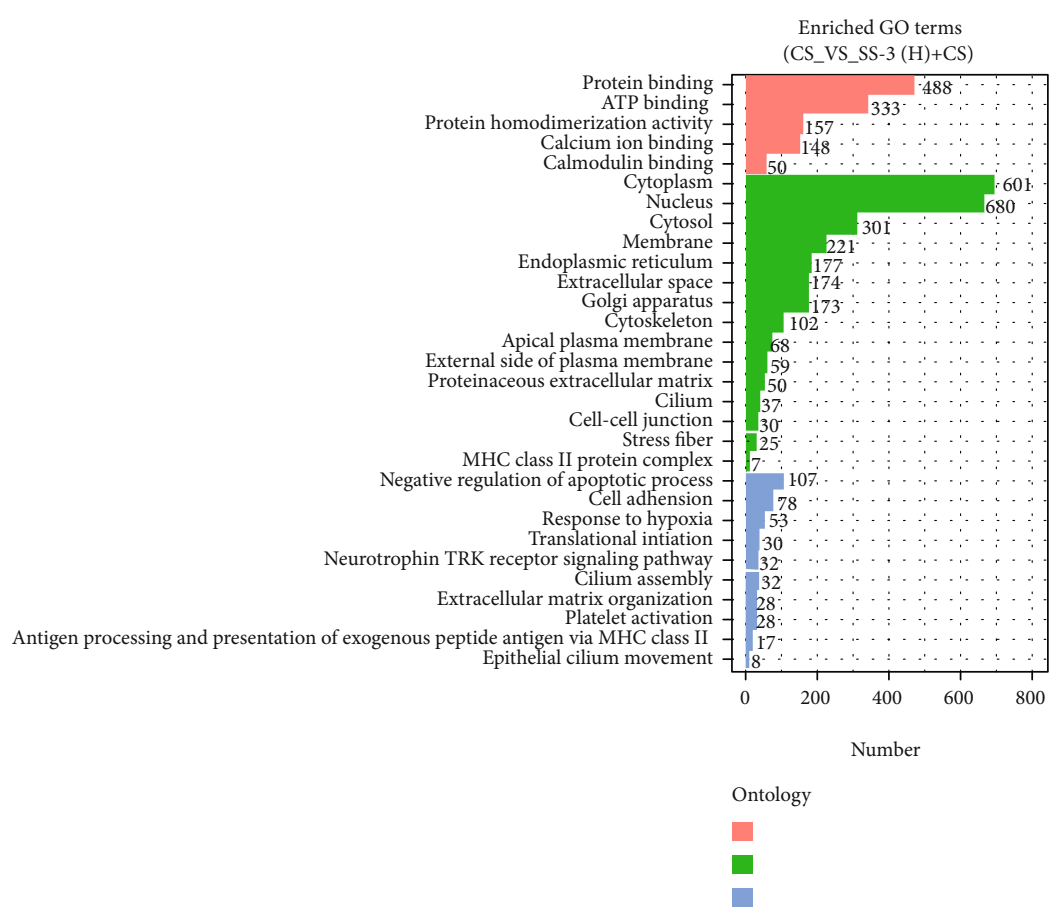

(b)

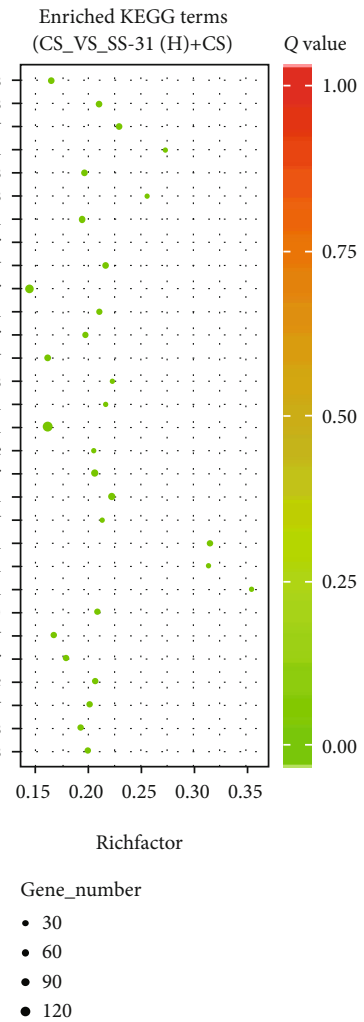

(c)

FIGURE 7: RNA sequencing in mouse lung. (a) Clustering of DEGs in the CS and SS-31 (H)+CS groups ( $n=3$ per group). (b) GO analysis of DEGs. The figure is composed of 3 parts: molecular functions, cellular components, and biological processes. The significance level of enrichment was set as a corrected $P$ value $<0.05$. (c) KEGG pathway analysis of DEGs. The bubble chart shows enrichment of DEGs in signaling pathways. The $y$-axis label represents the pathway, and the $x$-axis label represents the enrichment factor-i.e., the number of DEGs enriched in the pathway divided by the total number of genes in the background gene set. The size of each bubble represents the number of DEGs enriched in the pathway, and the color represents the significance of enrichment. Abbreviations: CS: cigarette smoke; DEG: differentially expressed gene; GO: Gene Ontology; KEGG: Kyoto Encyclopedia of Genes and Genomes; SS-31 (H): high-dose SS-31 (5 mg/kg). 


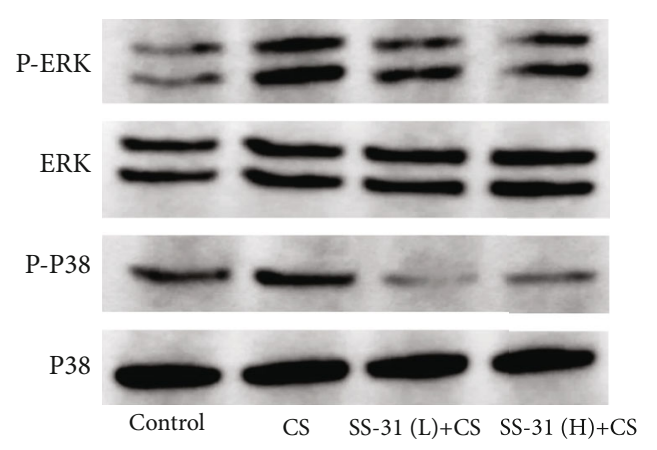

(a)

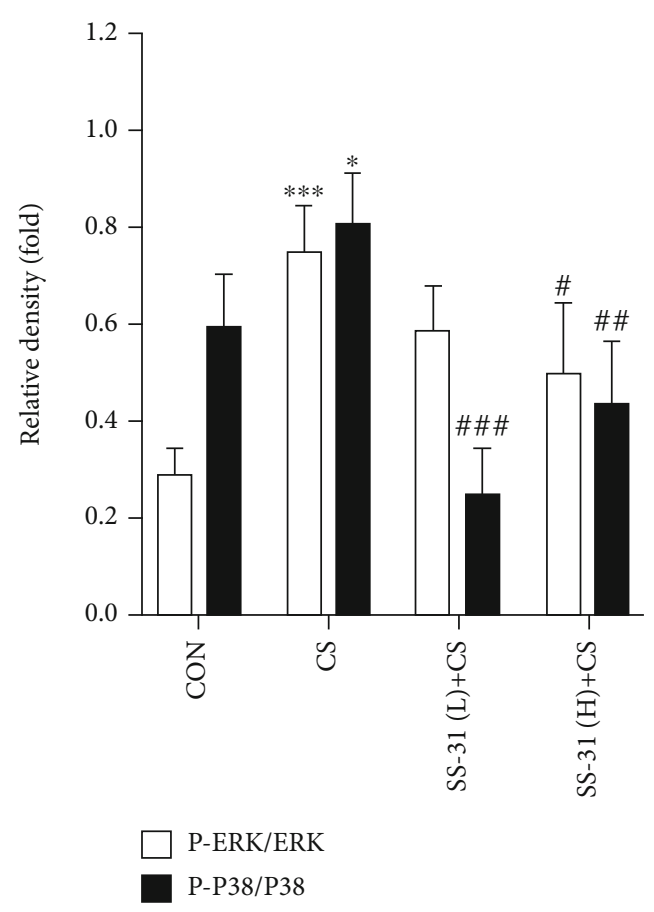

(b)

FIgURE 8: SS-31 alleviates CS-induced phosphorylation of ERK and P38. (a) Protein levels of ERK, P-ERK, P38, and P-P38 were detected by western blot. (b) Activation of these signaling molecules was evaluated based on the ratio of the density of phosphorylated protein to total protein ( $n=3$ per group). ${ }^{*} P<0.05$ and ${ }^{* * *} P<0.001$ vs. control; ${ }^{\#} P<0.05$, ${ }^{\# \#} P<0.01$, and ${ }^{\# \# \#} P<0.001$ vs. CS. Abbreviations: CS: cigarette smoke; ERK: extracellular signal-regulated kinase; P-ERK: phosphorylated extracellular signal-regulated kinase; P-P38: phosphorylated P38; SS-31 (H): high-dose SS-31 (5 mg/kg); SS-31 (L): low-dose SS-31 (2.5 mg/kg).

the lungs was positively correlated with neutrophil count (Supplementary Figure 1).

3.5. SS-31 Protects against Mitochondrial Dysfunction in CSExposed Mouse Lung. The levels of the mitochondrial fusion protein OPA1 and fission protein MFF were assayed as markers of mitochondrial function. CS exposure for 4 weeks reduced the expression of OPA1 and increased that of MFF in the lungs (Figures 6(a) and 6(b)). This was accompanied by a decrease in mitochondrial cytochrome $\mathrm{c}$ but an increase in the cytosolic pool (Figures 6(c) and 6(d)), which was reversed by high-dose SS-31 pretreatment. The western blot analysis and densitometry results are summarized in Supplementary Table 1.

3.6. RNA Sequencing Analysis of Mouse Lung. DEGs were defined as genes with a fold change $\geq 1.0$ in the SS-31 $(\mathrm{H})+\mathrm{CS}$ group compared to the CS group. According to this criterion, 4038 DEGs were identified (Figure 7(a)) including 2034 upregulated and 2004 downregulated genes; the top 10 of each are summarized in Supplementary Table 2. The DEGs were categorized into $30 \mathrm{GO}$ categories under 3 ontologies (Figure 7(b)) and 30 KEGG pathways (Figure $7(\mathrm{c})$ ). The KEGG pathway analysis revealed that these genes were enriched in the MAPK, cyclic (c) AMP, and $5^{\prime}$ AMP-activated protein kinase (AMPK) signaling pathways and extracellular matrix-receptor interaction (Figure 7(c)).
3.7. SS-31 Inhibits CS-Induced Phosphorylation of ERK and $P 38$. The signaling pathway potentially mediating the effects of SS-31 was investigated by evaluating the expression of proteins in mouse lung tissue samples by western blot. After 4 weeks of CS exposure, the phosphorylation of ERK and P38 was increased in mouse lung. High-dose SS-31 abrogated the activation of both proteins (Figure 8). The western blot analysis and densitometry results are summarized in Supplementary Table 1.

\subsection{SS-31 Prevents CSE-Induced Inflammatory Cytokine} Release via Inactivation of MAPK Signaling in Bronchial Epithelial Cells. To confirm the in vivo finding that SS-31 attenuated CS-induced inflammation and oxidative stress via inhibition of P38 MAPK signaling and to more closely examine the underlying mechanism, we evaluated the effects of anisomycin on BEAS-2B cells pretreated with CSE and SS31. We first assessed the toxicity of CSE with the CCK8 assay. The cell viability in cells treated with $2 \%, 4 \%, 6 \%$, and $8 \%$ CSE was $>80 \%$ compared to the control group, but $10 \%$ CSE considerably decreased the percentage of living cells $(<80 \%)$ (Figure 9(a)). To establish a bronchial epithelial cell model of CSE-induced airway inflammation, BEAS-2B cells were treated with $8 \%$ CSE for $24 \mathrm{~h}$ in the following experiments.

The P38 MAPK activator anisomycin was used to stabilize P38 at the protein level. Anisomycin significantly increased P38 phosphorylation (Figure 9(b)). SS-31 reduced the level of phosphorylated P38 MAPK in CSE-incubated 


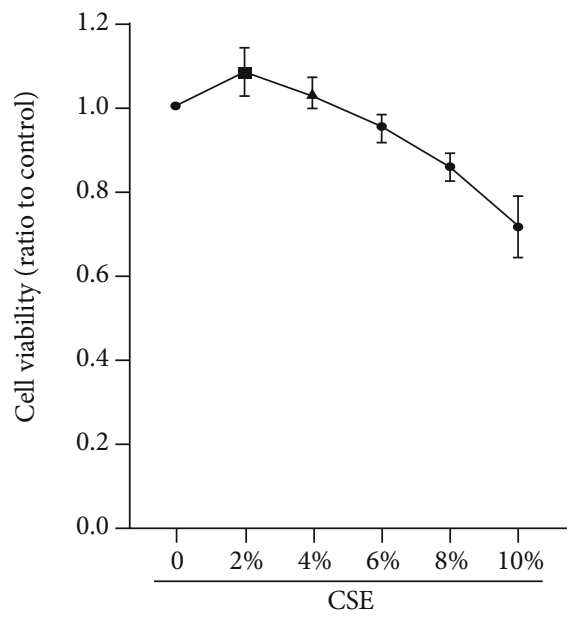

(a)

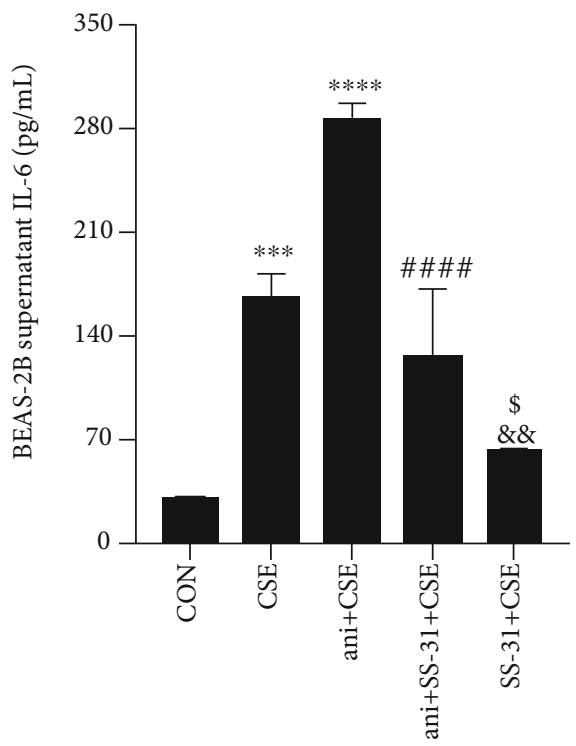

(d)

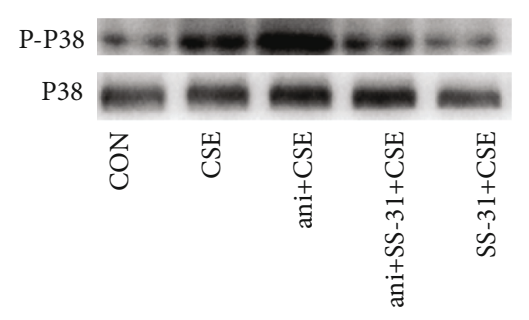

(b)

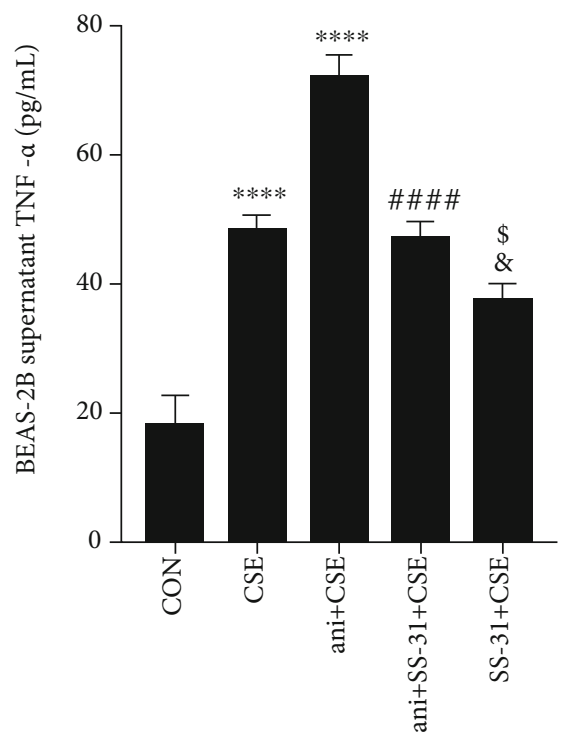

(e)

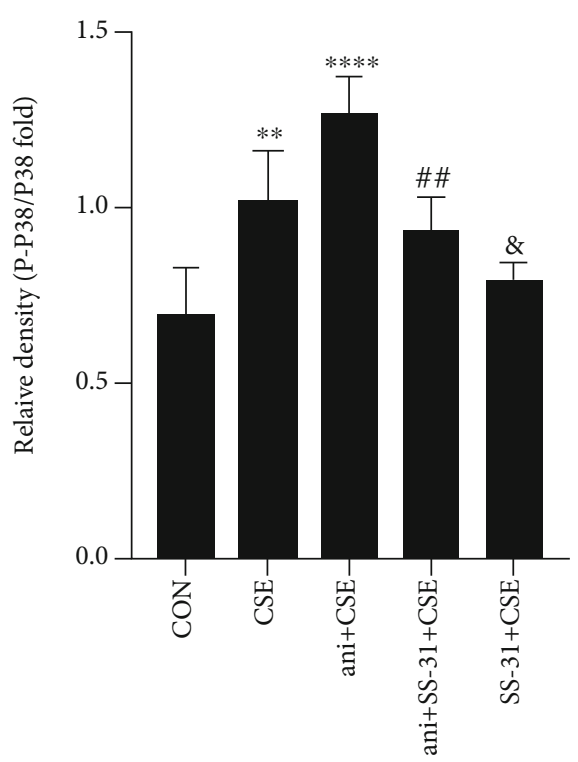

(c)

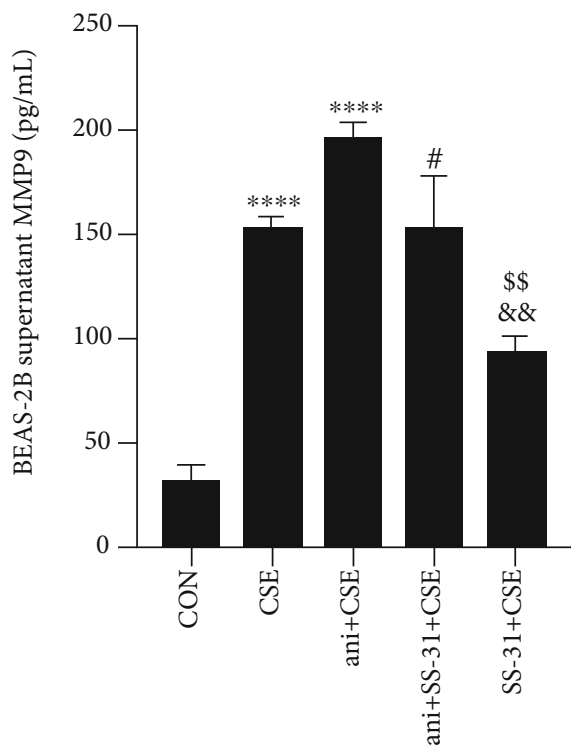

(f)

FIGURE 9: SS-31 inhibits CSE-induced inflammation and activation of MAPK signaling in BEAS-2B cells. (a) Cell viability was measured with CCK-8 following treatment with indicated concentrations of CSE ( $n=3$ per group). (b, c) Phosphorylated and total P38 protein levels ( $n=3$ per group). (d-f) IL-6 (d), TNF- $\alpha$ (e), and MMP9 (f) levels in BEAS-2B cell supernatant ( $n=3$ per group). ${ }^{* *} P<0.01$, ${ }^{* * *} P<0.001$, and

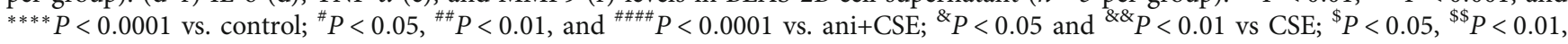
and ani+SS-31+CSE vs. SS-31+CSE. Abbreviations: ani: anisomycin; CSE: cigarette smoke extract; P-P38: phosphorylated P38.

BEAS-2B cells, but this was blocked by anisomycin (Figures 9(b) and 9(c)). Moreover, the levels of IL-6, TNF$\alpha$, and MMP9 were higher in the anisomycin+CSE+SS-31 group than in the SS-31+CSE group (Figures $9(\mathrm{~d})-9(\mathrm{f})$ ). These results imply that SS-31 inhibits MAPK signaling to alleviate CSE-induced inflammation.

3.9. SS-31 Prevents CSE-Induced Oxidative Stress via Inactivation of MAPK Signaling. ROS levels are an important indicator of intracellular oxidative stress. Anisomycin pretreatment reduced the suppressive effect of SS-31 on CSEinduced ROS production (Figure 10(a)) and abolished the downregulation of MDA activity and upregulation SOD activity in SS-31-treated BEAS-2B cells (Figures 10(b) and $10(c)$ ), although it had no effect on GSH-Px activity (Figure $10(\mathrm{~d})$ ). These results indicate that inactivation of MAPK signaling is essential for the protective effect of SS31 - which may be specific to antioxidant enzymes-against oxidative stress induced by CSE.

3.10. SS-31 Prevents CSE-Induced Mitochondrial Dysfunction via Inactivation of MAPK Signaling. The downregulation of OPA1 and upregulation of MFF induced by CSE in BEAS$2 \mathrm{~B}$ cells were reversed by SS-31, but these changes were 


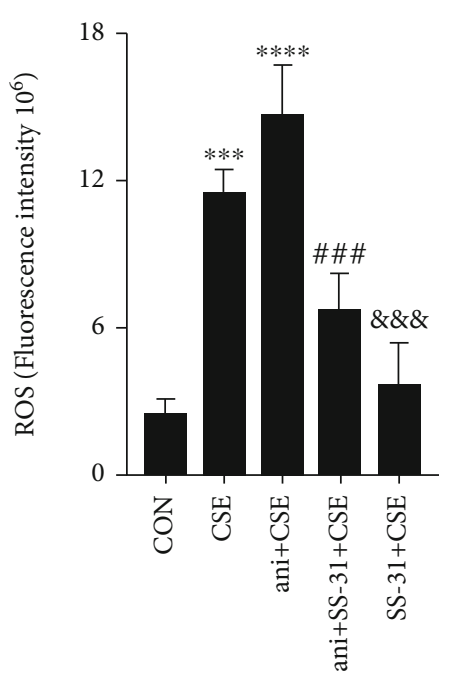

(a)

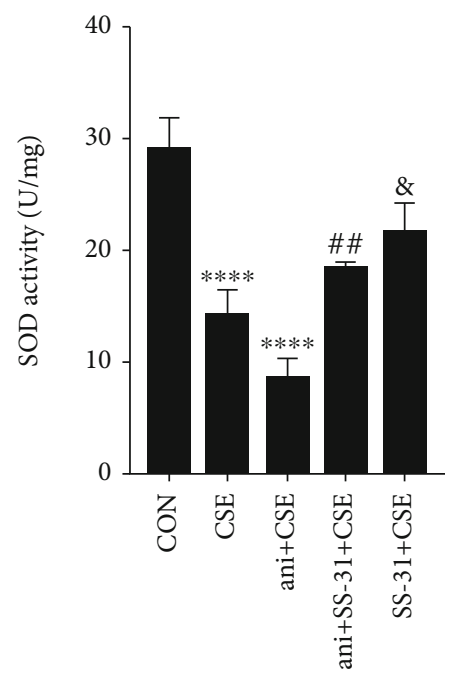

(c)

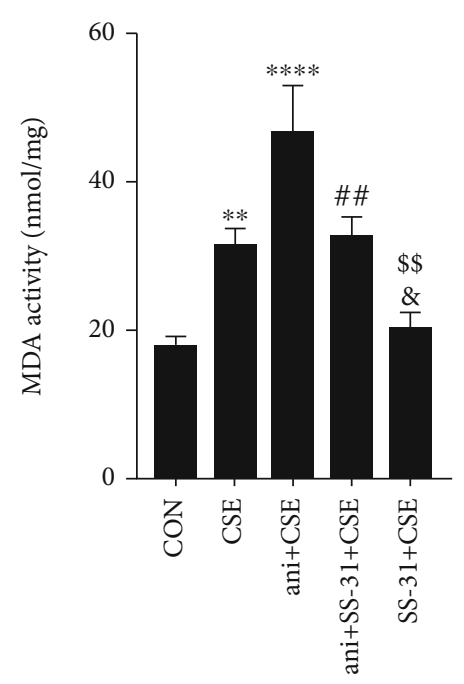

(b)

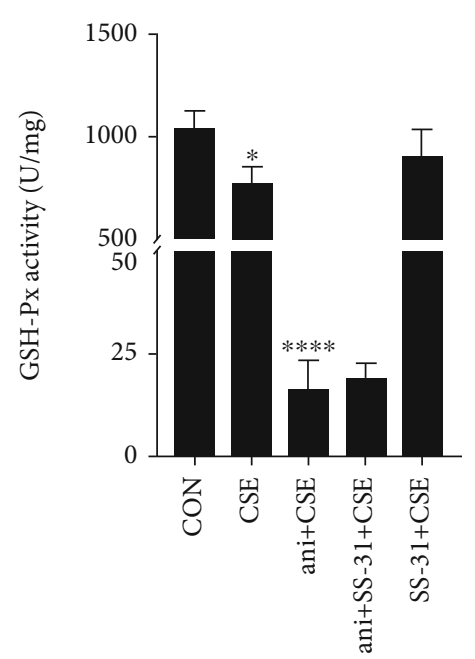

(d)

FIGURE 10: SS-31 suppresses CSE-induced oxidative stress via inhibition of MAPK signaling. (a) Intracellular ROS generation in BEAS-2B cells. (b-d) Activities of MDA (b), SOD (c), and GSH-Px (d) in cells ( $n=3$ per group). ${ }^{*} P<0.05,{ }^{* *} P<0.01,{ }^{* * *} P<0.001$, and ${ }^{* * * *} P<$ 0.0001 vs. control; ${ }^{\#} P<0.01$ and ${ }^{\# \# \#} P<0.001$ vs. ani+CSE; ${ }^{\&} P<0.05$ and ${ }^{\& \& \&} P<0.001$ vs. CSE; ${ }^{\$ \$} P<0.01$ and ani+SS-31+CSE vs. SS-31 +CSE. Abbreviations: ani: anisomycin; CSE: cigarette smoke extract; GSH-Px: glutathione peroxidase; MDA: malondialdehyde; ROS: intracellular reactive oxygen species; SOD: superoxide dismutase.

abolished by the MAPK activator anisomycin (Figures 11(a) and 11(b)). Thus, activation of the P38 MAPK signaling pathway can block the protective effects of SS-31 against CSE-induced mitochondrial dysfunction.

\section{Discussion}

SS-31 is a Szeto-Schiller peptide that selectively targets the inner mitochondrial membrane and exerts protective effects during inflammatory responses and oxidative stress [2527]. In the current study, we found that SS-31 attenuated CS-induced airway inflammation, mucus hypersecretion, and oxidative stress in mice. SS-31 also preserved mitochondrial function through up- and downregulation of mitochondrial proteins (OPA1 and MFF, respectively) and by blocking the release of cytochrome $\mathrm{c}$ into the cytosol. The results of the RNA sequencing analysis suggested that these effects may be related to inhibition of CS-induced MAPK signaling. In vitro experiments demonstrated that SS-31 protected BEAS-2B cells from CSE-induced inflammation, oxidative damage, and mitochondrial dysfunction via suppression of the MAPK signaling pathway.

CS-induced mitochondrial oxidative stress amplifies airway inflammation and airway mucus hypersecretion and is closely associated with COPD progression [28, 29]. Antioxidants are beneficial in COPD as they inhibit the inflammatory response [30]. SS-31 was shown to suppress inflammation, as evidenced by the decreased levels of IL-6, IL-1 $\beta[16,31]$, TNF- $\alpha$ [31], and MMP9 [14]. Additionally, SS-31 restored the activities of SOD, MDA [30], and MPA 


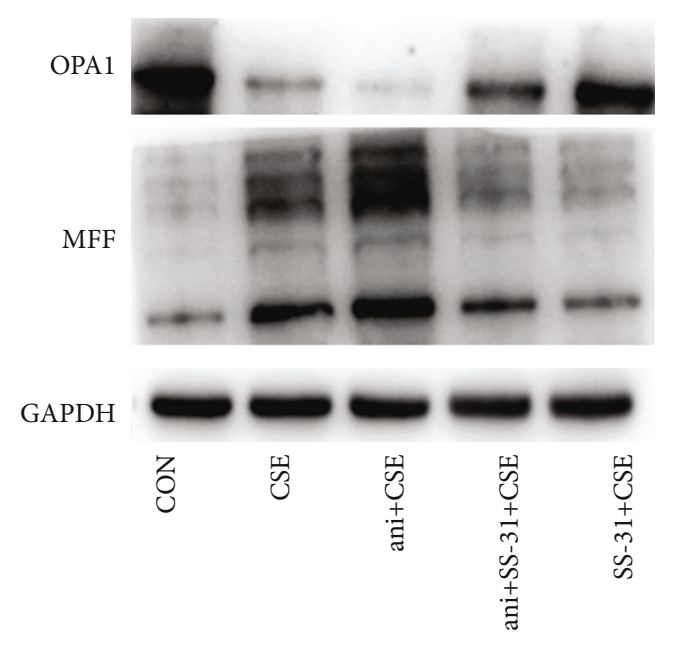

(a)

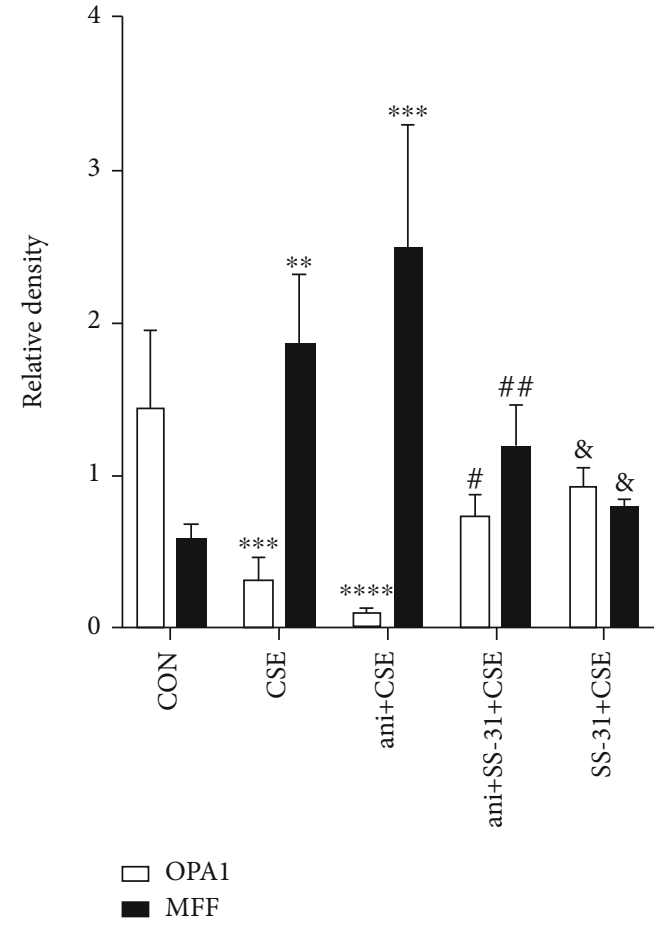

(b)

FIGURE 11: SS-31 protects against CSE-induced mitochondrial dysfunction via suppression of MAPK signaling. (a, b) Protein levels of OPA1 and MFF detected by western blot in BEAS-2B cells $\left(n=3\right.$ per group). ${ }^{* *} P<0.01,{ }^{* * *} P<0.001$, and ${ }^{* * * *} P<0.0001$ vs. control; ${ }^{\#} P<0.05$ and ${ }^{\#} P<0.01$ vs. ani+CSE; ${ }^{\circledR} P<0.05$ vs. CSE. Abbreviations: ani: anisomycin; CSE: cigarette smoke extract; MFF: mitochondrial fission factor; OPA1: optic atrophy 1.

[12] and mitigated ROS production [32], thereby balancing oxidative status. These results provide novel evidence that SS-31 has a protective role against CS-induced airway inflammation and oxidative stress and thus has therapeutic potential for the treatment of CS-related lung disorders such as COPD.

CS-induced mitochondrial dysfunction has been linked to the initiation and progression of COPD [8], which may be effectively treated with mitochondrial-targeting antioxidants [28]. SS-31 restored the balance of cytochrome c levels between mitochondria and the cytoplasm in renal fibrosis [33] and obstructive nephropathy [34]. Pretreatment with SS-31 also reversed alterations in the expression of the mitochondrial proteins dynamin-related protein (DRP) 1, FIS1, mitofusin (MFN) 1, MFN2, and OPA1 in Alzheimer disease [35]. Here, we demonstrate for the first time that SS-31 counters CS-induced airway inflammation and oxidative stress by alleviating mitochondrial dysfunction.

The RNA sequencing analysis revealed that SS-31 altered the expression of 4038 genes in mouse lung. Interestingly, one of the genes that was upregulated was the circadian clock gene nuclear receptor subfamily 1 group D member (NR1D) 1. The downregulation of NR1D1 has been linked to pathologic changes in the respiratory tract induced by CS [36]. Pretreatment with an NR1D1 agonist and antagonist blocked IL-1 $\beta$ secretion and increased macrophage and neutrophil infiltration, respectively [37], while mutation of the NR1D1 gene enhanced inflammation and chemokine release [38, 39]. NR1D1 regulates mitochondrial energy production and enhances cellular antioxidant mechanisms to protect cells against oxidative stress [40]. Thus, the modulation of mitochondrial oxidative stress and inflammation by SS-31 may involve disruption of the circadian clock, with NR1D1 serving as a downstream target of SS-31.

The RNA sequencing results suggested that SS-31 regulates many aspects of cell function including epithelial cilium movement and cilium assembly. CS exposure was shown to result in cilia loss and impaired beating [41], and alterations in cilia structure or function have been implicated in COPD pathogenesis [42]. Signaling molecules involved in the occurrence and development of COPD such as MAPK and CAMP help regulate inflammation and airway remodeling, as does extracellular matrix degradation [43]. AMPK modulates inflammatory responses, senescence, mitochondrial dysfunction, and metabolic dysregulation [44]. There is increasing evidence that constitutive or aberrant MAPK activation contributes to several COPD-associated phenotypes including mucus overproduction and secretion, inflammation, and cytokine expression $[45,46]$. Various small-molecule inhibitors may exert lung-protective effects by blocking the ERK1/2 and MAPK signaling $[43,47]$. It was previously reported that SS-31 exerts antioxidant effects by suppressing the activation of P38 MAPK [48-50]. This was confirmed by our observation that SS-31 treatment reversed the increases in ERK and P38 phosphorylation in CS-exposed mice. Thus, SS-31 protects the lungs in COPD by attenuating CS-induced activation of the ERK/P38 MAPK pathway, which was supported 
by the finding that the MAPK activator anisomycin [51] partly reversed the anti-inflammatory and antioxidant effects of SS-31 in response to CSE in bronchial epithelial cells.

The current study had several limitations. Firstly, in order to minimize the number of mice used, we did not include a control group that was pretreated with SS-31 but was not exposed to CS. Secondly, two BALF samples were not lavaged successfully in the CS and SS-31 (high)+CS groups; because of the small sample size, the inhibitory effect of $5 \mathrm{mg} / \mathrm{kg} \mathrm{SS}-31$ on the number of neutrophils in the BALF may have been minimized. Thirdly, because of a lack of specific equipment, we did not examine the effect of SS-31 treatment on lung function in mice exposed to CS. Finally, the effects of anisomycin and SS-31 on mitochondrial cytochrome $\mathrm{c}$ were not evaluated because of the difficulty of extracting this protein. A major challenge of studies on CSinduced inflammation is translating the experimental data into clinically relevant studies. For the clinical application of SS-31 in the treatment of CS-related disorders in humans, additional studies are needed to determine the appropriate dosing and potential adverse effects.

\section{Conclusion}

The results of this study demonstrate that SS-31 has therapeutic potential for the treatment of CS-induced lung diseases-particularly COPD-based on its anti-inflammatory and antioxidant properties in vitro and in vivo, which involve the downregulation of MAPK signaling and modulation of mitochondrial function.

\section{Data Availability}

Data used to support the findings of this study are available from the corresponding author upon request.

\section{Disclosure}

The funders had no role in study design, data collection and analysis, decision to publish, or preparation of the manuscript.

\section{Conflicts of Interest}

The authors declare no conflict of interest.

\section{Authors' Contributions}

YCS and FQW provided funding and designed the experiments. DQY and LJG performed the experiments and interpreted the data. QNZ and TW analyzed the data. CW and LC contributed reagents/materials/analysis tools. DQY, QNZ, and TW wrote the first draft of the paper. YCS and FQW critically edited the manuscript and supervised the study. De-qing Yang, Qiu-nan Zuo, and Tao Wang contributed equally to this work.

\section{Acknowledgments}

This work was supported by grants from the National Natural Science Foundation of China (31871157 and 81830001), National Key Research and Development Program in China (2016YFC0901102 and 2016YFC1304500), and Sichuan Key Research and Development Program (2019YFS0232).

\section{Supplementary Materials}

Supplementary Figure 1: correlation between MPO activity and the number of neutrophils in the lungs was analyzed. Supplementary Table 1: all western blot images with densitometry are summarized. Supplementary Table 2: the top 10 upregulated and downregulated DEGs identified by RNA sequencing analysis. (Supplementary Materials)

\section{References}

[1] A. Agusti and R. Faner, "Chronic obstructive pulmonary disease pathogenesis," Clinics in Chest Medicine, vol. 41, no. 3, pp. 307-314, 2020.

[2] B. Wang, D. Xiao, and C. Wang, "Smoking and chronic obstructive pulmonary disease in Chinese population: a meta-analysis," The Clinical Respiratory Journal, vol. 9, no. 2, pp. 165-175, 2015.

[3] M. Zhou, H. Wang, X. Zeng et al., "Mortality, morbidity, and risk factors in China and its provinces, 1990-2017: a systematic analysis for the Global Burden of Disease Study 2017," Lancet, vol. 394, no. 10204, pp. 1145-1158, 2019.

[4] D. Singh, A. Agusti, A. Anzueto et al., "Global strategy for the diagnosis, management, and prevention of chronic obstructive lung disease: the GOLD science committee report 2019," The European Respiratory Journal, vol. 53, no. 5, p. 1900164, 2019.

[5] M. Hikichi, K. Mizumura, S. Maruoka, and Y. Gon, "Pathogenesis of chronic obstructive pulmonary disease (COPD) induced by cigarette smoke," Journal of Thoracic Disease, vol. 11, no. S17, pp. S2129-S2140, 2019.

[6] G. Caramori, P. Kirkham, A. Barczyk, A. Di Stefano, and I. Adcock, "Molecular pathogenesis of cigarette smokinginduced stable COPD," Annals of the New York Academy of Sciences, vol. 1340, no. 1, pp. 55-64, 2015.

[7] M. Aghapour, A. H. V. Remels, S. D. Pouwels et al., "Mitochondria: at the crossroads of regulating lung epithelial cell function in chronic obstructive pulmonary disease," American Journal of Physiology Lung Cellular and Molecular Physiology, vol. 318, no. 1, pp. L149-1164, 2020.

[8] S. W. Ryter, I. O. Rosas, C. A. Owen et al., "Mitochondrial dysfunction as a pathogenic mediator of chronic obstructive pulmonary disease and idiopathic pulmonary fibrosis," Annals of the American Thoracic Society, vol. 15, Supplement_4, pp. S266-s272, 2018.

[9] J. D. Chavez, X. Tang, M. D. Campbell et al., "Mitochondrial protein interaction landscape of SS-31," Proceedings of the National Academy of Sciences of the United States of America, vol. 117, no. 26, pp. 15363-15373, 2020.

[10] Y. Mo, S. Deng, L. Zhang et al., "SS-31 reduces inflammation and oxidative stress through the inhibition of Fis1 expression in lipopolysaccharide-stimulated microglia," Biochemical and Biophysical Research Communications, vol. 520, no. 1, pp. 171-178, 2019. 
[11] I. Escribano-Lopez, N. Diaz-Morales, F. Iannantuoni et al., "The mitochondrial antioxidant SS-31 increases SIRT1 levels and ameliorates inflammation, oxidative stress and leukocyte-endothelium interactions in type 2 diabetes," Scientific Reports, vol. 8, no. 1, p. 15862, 2018.

[12] L. L. Zhu, M. Q. Li, F. He, S. B. Zhou, and W. Jiang, "Mitochondria targeted peptide attenuates mitochondrial dysfunction, controls inflammation and protects against spinal cord injury-induced lung injury," Cellular Physiology and Biochemistry, vol. 44, no. 1, pp. 388-400, 2018.

[13] D. Yang, D. Xu, T. Wang et al., "Mitoquinone ameliorates cigarette smoke-induced airway inflammation and mucus hypersecretion in mice," International Immunopharmacology, vol. 90, article 107149, 2021.

[14] H. I. Lu, T. H. Huang, P. H. Sung et al., "Administration of antioxidant peptide SS-31 attenuates transverse aortic constriction-induced pulmonary arterial hypertension in mice," Acta Pharmacologica Sinica, vol. 37, no. 5, pp. 589603, 2016.

[15] Y. Zhu, H. Wang, J. Fang et al., "SS-31 provides neuroprotection by reversing mitochondrial dysfunction after traumatic brain injury," Oxidative Medicine and Cellular Longevity, vol. 2018, Article ID 4783602, 12 pages, 2018.

[16] J. Cai, Y. Jiang, M. Zhang et al., "Protective effects of mitochondrion-targeted peptide SS-31 against hind limb ischemia-reperfusion injury," Journal of Physiology and Biochemistry, vol. 74, no. 2, pp. 335-343, 2018.

[17] D. Li, D. Xu, T. Wang et al., "Silymarin attenuates airway inflammation induced by cigarette smoke in mice," Inflammation, vol. 38, no. 2, pp. 871-878, 2015.

[18] H. Wang, T. Yang, T. Wang et al., "Phloretin attenuates mucus hypersecretion and airway inflammation induced by cigarette smoke," International Immunopharmacology, vol. 55, pp. 112-119, 2018.

[19] X. Sun and W. Zhang, "Silencing of Gal-7 inhibits TGF- $\beta_{1}$ induced apoptosis of human airway epithelial cells through JNK signaling pathway," Experimental Cell Research, vol. 375, no. 2, pp. 100-105, 2019.

[20] S. K. Yang, Y. C. Han, J. R. He et al., "Mitochondria targeted peptide SS-31 prevent on cisplatin-induced acute kidney injury via regulating mitochondrial ROS-NLRP3 pathway," Biomedicine \& Pharmacotherapy, vol. 130, article 110521, 2020.

[21] D. Li, J. Hu, T. Wang et al., "Silymarin attenuates cigarette smoke extract-induced inflammation via simultaneous inhibition of autophagy and ERK/p38 MAPK pathway in human bronchial epithelial cells," Scientific Reports, vol. 6, no. 1, article 37751, 2016.

[22] E. J. Park, Y. J. Park, S. J. Lee, K. Lee, and C. Yoon, "Whole cigarette smoke condensates induce ferroptosis in human bronchial epithelial cells," Toxicology Letters, vol. 303, pp. 55-66, 2019.

[23] Y. Xin, C. Li, Y. Guo, R. Xiao, H. Zhang, and G. Zhou, "RNASeq analysis reveals a negative role of MSMO1 with a synergized NSDHL expression during adipogenesis of 3T3-L1," Bioscience, Biotechnology, and Biochemistry, vol. 83, no. 4, pp. 641-652, 2019.

[24] M. A. Harris, J. Clark, A. Ireland et al., "The Gene Ontology (GO) database and informatics resource," Nucleic Acids Research, vol. 32, no. 90001, pp. 258D-2261, 2004.

[25] H. H. Szeto, "First-in-class cardiolipin-protective compound as a therapeutic agent to restore mitochondrial bioenergetics,"
British Journal of Pharmacology, vol. 171, no. 8, pp. 20292050, 2014.

[26] H. H. Szeto, "Stealth peptides target cellular powerhouses to fight rare and common age-related diseases," Protein and Peptide Letters, vol. 25, no. 12, pp. 1108-1123, 2019.

[27] D. F. Dai, Y. A. Chiao, D. J. Marcinek, H. H. Szeto, and P. S. Rabinovitch, "Mitochondrial oxidative stress in aging and healthspan," Longev Healthspan, vol. 3, no. 1, p. 6, 2014.

[28] P. J. Barnes, "Oxidative stress-based therapeutics in COPD," Redox Biology, vol. 33, p. 101544, 2020.

[29] Y. Xu, H. Liu, and L. Song, "Novel drug delivery systems targeting oxidative stress in chronic obstructive pulmonary disease: a review," Journal of Nanobiotechnology, vol. 18, no. 1, p. 145, 2020.

[30] K. F. Chung and J. A. Marwick, "Molecular mechanisms of oxidative stress in airways and lungs with reference to asthma and chronic obstructive pulmonary disease," Annals of the New York Academy of Sciences, vol. 1203, no. 1, pp. 85-91, 2010.

[31] Y. Liu, W. Yang, X. Sun et al., "SS31 ameliorates sepsisinduced heart injury by inhibiting oxidative stress and inflammation," Inflammation, vol. 42, no. 6, pp. 2170-2180, 2019.

[32] S. Petri, M. Kiaei, M. Damiano et al., "Cell-permeable peptide antioxidants as a novel therapeutic approach in a mouse model of amyotrophic lateral sclerosis," Journal of Neurochemistry, vol. 98, no. 4, pp. 1141-1148, 2006.

[33] H. Zhao, Y. J. Liu, Z. R. Liu et al., "Role of mitochondrial dysfunction in renal fibrosis promoted by hypochlorite- modified albumin in a remnant kidney model and protective effects of antioxidant peptide SS-31," European Journal of Pharmacology, vol. 804, pp. 57-67, 2017.

[34] Z. R. Liu, S. Q. Chen, Y. W. Zou et al., "Hypochlorite modified albumins promote cell death in the tubule interstitium in rats via mitochondrial damage in obstructive nephropathy and the protective effects of antioxidant peptides," Free Radical Research, vol. 52, no. 5, pp. 616-628, 2018.

[35] P. H. Reddy, M. Manczak, and R. Kandimalla, "Mitochondriatargeted small molecule SS31: a potential candidate for the treatment of Alzheimer's disease," Human Molecular Genetics, vol. 26, no. 8, pp. 1483-1496, 2017.

[36] V. T. Vasu, C. E. Cross, and K. Gohil, "Nr1d1, an important circadian pathway regulatory gene, is suppressed by cigarette smoke in murine lungs," Integrative Cancer Therapies, vol. 8, no. 4, pp. 321-328, 2009.

[37] D. Yu, X. Fang, Y. Xu et al., "Rev-erb $\alpha$ can regulate the NF$\kappa \mathrm{B} / \mathrm{NALP} 3$ pathway to modulate lipopolysaccharide- induced acute lung injury and inflammation," International Immunopharmacology, vol. 73, pp. 312-320, 2019.

[38] I. K. Sundar, K. Rashid, M. T. Sellix, and I. Rahman, "The nuclear receptor and clock gene REV-ERB $\alpha$ regulates cigarette smoke-induced lung inflammation," Biochemical and Biophysical Research Communications, vol. 493, no. 4, pp. 1390-1395, 2017.

[39] M. Pariollaud, J. E. Gibbs, T. W. Hopwood et al., "Circadian clock component REV-ERB $\alpha$ controls homeostatic regulation of pulmonary inflammation," The Journal of Clinical Investigation, vol. 128, no. 6, pp. 2281-2296, 2018.

[40] S. Sengupta, G. Yang, J. C. O’Donnell et al., "The circadian gene Rev-erb $\alpha$ improves cellular bioenergetics and provides preconditioning for protection against oxidative stress," Free Radical Biology \& Medicine, vol. 93, pp. 177-189, 2016. 
[41] S. M. Simet, J. H. Sisson, J. A. Pavlik et al., "Long-term cigarette smoke exposure in a mouse model of ciliated epithelial cell function," American Journal of Respiratory Cell and Molecular Biology, vol. 43, no. 6, pp. 635-640, 2010.

[42] J. E. Peabody, R. J. Shei, B. M. Bermingham et al., "Seeing cilia: imaging modalities for ciliary motion and clinical connections," American Journal of Physiology. Lung Cellular and Molecular Physiology, vol. 314, no. 6, pp. L909-1921, 2018.

[43] C. Wang, J. Zhou, J. Wang et al., "Progress in the mechanism and targeted drug therapy for COPD," Signal Transduction and Targeted Therapy, vol. 5, no. 1, p. 248, 2020.

[44] Z. Zhang, X. Cheng, L. Yue et al., "Molecular pathogenesis in chronic obstructive pulmonary disease and therapeutic potential by targeting AMP-activated protein kinase," Journal of Cellular Physiology, vol. 233, no. 3, pp. 1999-2006, 2018.

[45] B. A. Mercer and J. M. D'Armiento, "Emerging role of MAP kinase pathways as therapeutic targets in COPD," International Journal of Chronic Obstructive Pulmonary Disease, vol. 1, no. 2, pp. 137-150, 2006.

[46] G. C. A. Manley, L. C. Parker, and Y. Zhang, "Emerging regulatory roles of dual-specificity phosphatases in inflammatory airway disease," International Journal of Molecular Sciences, vol. 20, no. 3, p. 678, 2019.

[47] A. Banerjee, C. Koziol-White, and R. Panettieri Jr., "p38 MAPK inhibitors, IKK2 inhibitors, and TNF $\alpha$ inhibitors in COPD," Current Opinion in Pharmacology, vol. 12, no. 3, pp. 287-292, 2012.

[48] Y. Hou, S. Li, M. Wu et al., "Mitochondria-targeted peptide SS31 attenuates renal injury via an antioxidant effect in diabetic nephropathy," American Journal of Physiology. Renal Physiology, vol. 310, no. 6, pp. F547-F559, 2016.

[49] D. F. Dai, T. Chen, H. Szeto et al., "Mitochondrial targeted antioxidant peptide ameliorates hypertensive cardiomyopathy," Journal of the American College of Cardiology, vol. 58, no. 1, pp. 73-82, 2011.

[50] Y. Mizuguchi, J. Chen, S. V. Seshan, D. P. Poppas, H. H. Szeto, and D. Felsen, "A novel cell-permeable antioxidant peptide decreases renal tubular apoptosis and damage in unilateral ureteral obstruction," American Journal of Physiology-Renal Physiology, vol. 295, no. 5, pp. F1545-F1553, 2008.

[51] C. Rebeyrol, V. Saint-Criq, L. Guillot et al., "Glucocorticoids reduce inflammation in cystic fibrosis bronchial epithelial cells," Cellular Signalling, vol. 24, no. 5, pp. 1093-1099, 2012. 